\title{
BIOINDICATION USING DIVERSITY AND ECOLOGY OF ALGAE OF THE ALAKOL LAKE, KAZAKHSTAN
}

\author{
JiYenbeKov, A. ${ }^{1}$ - BARINOVA, S..$^{2 *}$ - Bigaliev, A. ${ }^{1}$ - NuRASHOV, S. ${ }^{3}$ - SAMETOVA, E. ${ }^{3}-$ \\ FAHIMA, T. ${ }^{2}$ \\ ${ }^{1}$ Al-Faraby Kazakh National University, Almaty 050040, Kazakhstan \\ (e-mail:zh-ai-bek@mail.ru; aitkhazha@gmail.com) \\ ${ }^{2}$ Institute of Evolution, University of Haifa \\ Mount Carmel, 199 Abba Khoushi Ave., Haifa 3498838, Israel \\ (e-mail:tfahima@evo.haifa.ac.il; phone: +972-4-824-9799; fax: +972-4-828-8313) \\ ${ }^{3}$ Institute of Botany and Phytointroduction, Almaty 050040, Kazakhstan \\ (e-mail:nurashs@mail.ru; elyasam@mail.ru) \\ *Corresponding author \\ e-mail: sophia@evo.haifa.ac.il \\ (Received $31^{\text {st }}$ Jul 2018; accepted $8^{\text {th }}$ Oct 2018)
}

\begin{abstract}
The aim of present study were to reveal species-indicators of the Alakol Lake communities and assess the water quality with bioindication and statistical methods. Algal communities in the Alakol Lake Natural State Reserve were studied in 21 samples collected during August 2015-2017 summer field trips. Altogether 208 algal species from five taxonomic Divisions were indicators of ten water properties: temperature, oxygenation, organic pollution, salinity, trophic state of the water, and nutrition type of algal species. It was the first experience of the bioindicational approach implementation for ecological assessment of water quality in the Alakol Lake. Diatom species, which are most indicative, strongly prevailed in the three studied areas of the lake. We revealed that algal species can characterize the water of the lake as low alkaline, low saline, temperate, middle oxygenated water with low-to middle organic pollution that comes from Koktuma and Kamyskala areas and decrease from north to south. The lake tropic state and intensity of self-purification are also increase from north to south. Algal communities aremostly represented by periphytonic and benthic autotrophic species. Statistical analysis of speciesenvironment relationships revealed that most of species preferred to survive in communities with complicated structure which formed in low organically polluted waters. Bioindication assess the Alakol Lake as oligotrophic and meso-eutrophic, of Class 2 and 3 of Water Quality with high level of selfpurification capacity.
\end{abstract}

Keywords: algae, bioindication, organic pollution, salinity, Alakol Lake, Kazakhstan

\section{Introduction}

The problem of water quality monitoring in developing countries there are concentrated in a poorly developed network of stations and low provision with instruments and specialists, such as in Kazakhstan. As a result, many water bodies, which could be sources of water supply to the population and the economy, remain unexplored. More often, some hydrophysical and hydrochemical parameters are determined on the water body, and the biotic part of the water ecosystem remains weakly explored or unexplored at all. In such cases, bioindication methods are of particular value, since they help not only to assess the state of the ecosystem of the water body, but also to forecast its development. Kazakhstan is a significant territory located in the semi-arid climate zone, where it is especially important to have an understanding of water quality in water bodies. There are some large lakes in this region 
as the Caspian Sea, Aral Sea, Balkhash, Issyk-Kul Lake and Alakol Lake. Despite this, there are still large unexplored water bodies on the territory of Kazakhstan, such as the Alakol Lake.

Our interest in bioindication research of lakes in the territory of Kazakhstan arose about 20 years ago. Lakes were studied in protected natural areas (Barinova et al., 2002; Barinova et al., 2011; Krupa et al., 2016) and a comparison was made of the floral composition of algae with lakes on the climatically similar territory of Israel (Barinova et al., 2009). In all these cases, bioindication on algae helped to identify the main properties of lake ecosystems in conditions of semi-arid climate, such as salinity and drying. In addition, Lake Balkhash was investigated with the help of bioindication on algae as one of the largest lakes in the semi-arid climatic zone in the territory of Kazakhstan (Krupa et al., 2014; Barinova et al., 2017; Krupa et al., 2017a,b,c; Barinova et al., 2018a,b). Bioindication methods proved to be particularly effective in the study of lakes, reservoirs and rivers on the territory of Kazakhstan that are subject to anthropogenic pollution (Barinova and Krupa, 2017a,b; Barinova et al., 2018a; Krupa et al., 2017d, 2018a,b). Especial attention can be given to the lakes in the Natural Reserves as, for example, the Alakol Lake.

The Alakol Lake is located on the Balkhash-Alakol lowland on the border of the Almaty and East Kazakhstan regions. The lake is representing the eastern part of the Balkhash-Alakol Basin. More than 15 tributaries including main of them the rivers Urzhar, Katynsu, Emelkysa, Ygrajty, Zhamanty, Zhamanotkel, and Tasty, which are permanently dry in the summer period because of semi-arid climate impact are input into lake. The Parliament of Kazakhstan has ratified the Convention on Biological Diversity in 1994. One of the important steps to affirming its desire to preserve the unique richness of nature and the implementation of these documents was the creation in 1998 of the Alakol Lake State Reserve. The identification of the biological diversity was the first aim of investigation of the new natural reserve. Up to now, only some aquatic invertebrates were studied accidentally (Krupa et al., 2010), but the algal diversity study is in initial step because up to current research the information about algal species of the Alakol Lake was absent. During our study in 2015-2017, we revealed 203 species and infraspecies of algae in the Alakol Lake communities (Jiyenbekov et al., 2018).

The aim of present study was to reveal species-indicators from the list of algae that has been found by us in the Alakol Lake and assess the water quality and major influencing parameters with bioindication and statistical methods.

\section{Study site description}

Lake Alakol and the adjacent part of its catchment basin is a protected natural area. Studied lake is represent high-mineralized drainage lake, belongs to the ecoregion 624 (Balkash - Alakul) according to the FEOW classification (Freshwater Ecoregions of the World). The Alakol Lake is located at an altitude of $348 \mathrm{~m}$ above sea level and earlier was a part of a single water system with Lake Balkhash (Berezovikov, 2006). Both lakes represent a drainless catchment basin in the eastern part of the Balkhash-Alakol depression (Fig. 1).

The area of the lake surface with the islands is about $2,696 \mathrm{~km}^{2}$. The length of the coastline is about $348 \mathrm{~km}$. The total volume of water is about $58.56 \mathrm{~km}^{3}$. The lake have oval shape with length $104 \mathrm{~km}$, and width about $52 \mathrm{~km}$. Average depth is about $22 \mathrm{~m}$ with the greatest is $54 \mathrm{~m}$. The Alakol Lake and close placed lakes Sasykkol, Uyaly, 
Zhalanashkol and others smaller, together are formed the Alakol Lake system. There are few islands Ulken, Kishkeni Araltobe, Belkuduk, etc. that are placed in the center of the Alakol Lake. This area have sharply continental climate. A complex wind regime is observed above the lake with maximum wind speed over the northern parts of the lake is about 40-50 m per second, over the southeastern and central parts about 50-60 m per second. The most active winds are in the autumn-winter period, when the wave height in the lake surface can be up to 2-2.5 m (Berezovikov, 2006).

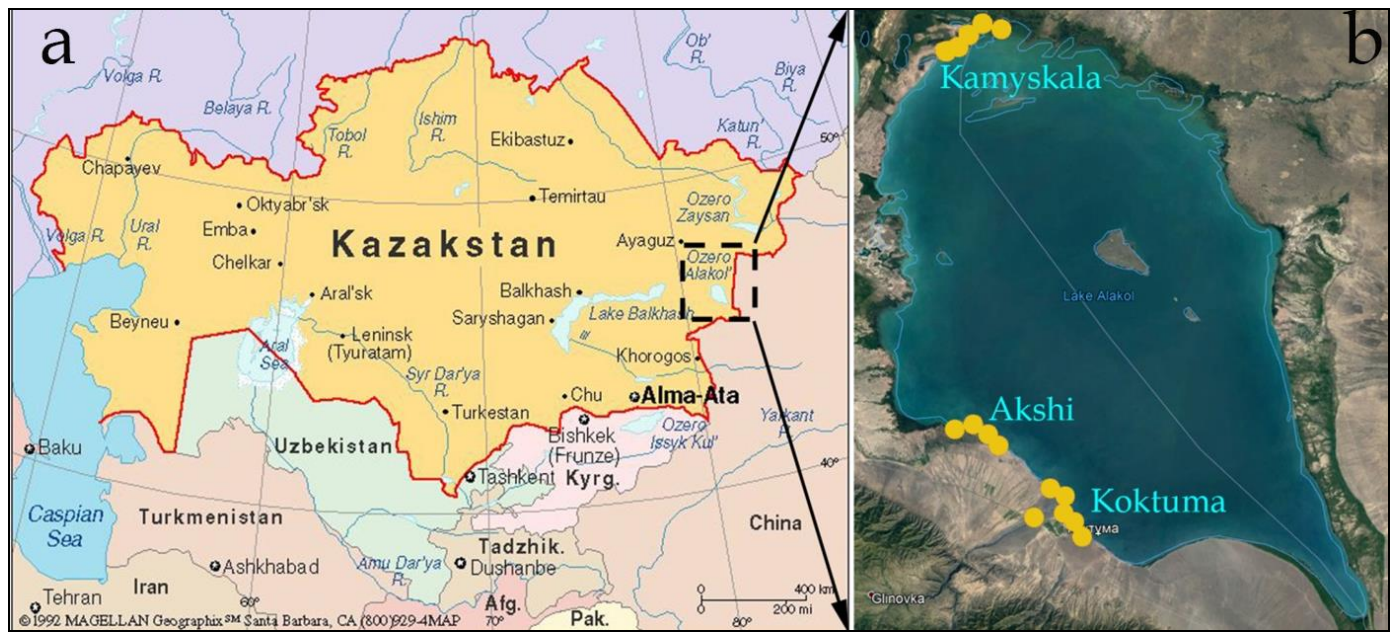

Figure 1. The Alakol Lake sampling points (yellow) in three sampling areas Kamyskala, Akshi, and Koktuma in 2015-2017

The lake have ice-cover period about 2 months (February-March). The largest ice thickness is about $0.8 \mathrm{~m}$ (in February) with melting period in April-early May. The water temperature reaches $7-15^{\circ} \mathrm{C}$ in late May and is higher in July-August when the winds are lower. The waters of the lake are brackish with chloride-sodium and chloridesulfate-sodium composition. Total dissolved solids (TDS) concentration is varied between 0.8 and $9.5 \mathrm{~g} \mathrm{l}^{-1}$ depending on the lake area with averaging about 5.9-7.8 $\mathrm{g} \mathrm{l}^{-1}$ but it was rather high due to sulfates (Krupa et al., 2010). High content of fluorine and bromine are in the waters of Lake Alakol (Berezovikov, 2006).

\section{Materials and methods}

\section{Sampling}

A total 21 samples of algae from microphytobenthos and phytoplankton were collected during August 2015-2017 summer field trips from three areas of the Alakol Lake (Fig. 1). Phytoplankton samples were taken with the Apstein net, pore diameter of $20 \mu \mathrm{m}$. Microphytobenthos was collected by scraping from the surfaces of stones, plants, and from the bottom. Samples of charophytes were collected as whole plants with rhizoids and partly herbarized, and partly fixed in $4 \%$ of neutral formaldehyde of ethanol. The microphytobenthos and phytoplankton samples were fixed in place immediately after sampling by $4 \%$ neutral formaldehyde solution, and some of them with $96 \%$ ethanol. Meteorological conditions of the water (swell, visual assessment), air (temperature, by termometer), water temperature (termometer), and geographic 
coordinates of sampling points (with Garmin GPSMAP 64 equipment) were determined along the collection of algae. The water transparency was determined with the Secchi disk and water $\mathrm{pH}$ with universal indicator paper in the sampling points.

Collected samples were transported to the laboratory of the Institute of Botany and Phytointroduction, Almaty, in the icebox, and part of fixed samples to the Laboratory of algal diversity and ecology, Institute of Evolution, University of Haifa, Israel.

\section{Laboratory study}

Algal samples were studied in three repetitions under light microscopes MBI-3, Amplival, and Carl Zeiss Axioskop-40 with high-resolution digital camera AxioCam MRc-5 and the AxioVision 4.8 program under the 600x-1000x magnification. The cells size of the studied species was obtained by using an ocular micrometer at the Institute of Botany and Phytointroduction, Almaty. Samples were processed at the Institute of Evolution, University of Haifa, Israel, under light microscope M4000-D at magnifications 400x - 1000x also. The glass slides of diatom algae were made with peroxide technique (Barinova, 1988; Barinova, 2017a) in the Eliashev media and Canadian balsam.

The images of defined algal species were made under microscope with digital camera OMAX A35100U and Motic MBI-300.

Saprobity index S (Sládeček, 1973, 1986) was calculated with species-specific indices and abundance of identified species for each algal community as:

$$
S=\sum_{i=1}^{n}\left(s_{i} \times a_{i}\right) / \sum_{i=1}^{n}\left(a_{i}\right)
$$

where: $\mathrm{S}$ - Saprobity index of algal community; $\mathrm{s}_{i}-$ species-specific Saprobity index; $\mathrm{a}_{i}$ - species abundance. The abundance of each species cells in the samples and slides was assessed with 6-score scale (Barinova et al., 2006; Barinova, 2017a).

The international handbooks were used for the species composition determination. The modern names of taxa were unified with help of algaebase.org (Guiry and Guiry, 2018) and organized in accordance with the nomenclature of Cavalier-Smith (2004).

Ecological indicator properties of defined species were assessed with help of our database (Barinova et al., 2006). Standard deviation (stdev) and polynomial trend lines were constructed with help of the Microsoft Excel program. Shannon Index of diversity was calculated in the GRAPHS program (Novakovsky, 2004). Statistically generated 3D surface plots were constructed with Distance Weighted Least Squares method in Statistica 12.0 program. Canonical correspondence analysis (CCA) was doing in the CANOCO statistical program for Windows 4.5 package (Ter Braak and Šmilauer, 2002).

\section{Results and Discussion}

Sampling points and areas with geographical coordinates are given in Table 1 with total species richness, Shannon Index, and Saprobity index $\mathrm{S}$ (Eq. 1). The water temperature was about $22^{\circ} \mathrm{C}-23^{\circ} \mathrm{C}$, and the water $\mathrm{pH}$ was about 7.5 in the sampling points during August of 2015-2017 field trips. 
Table 1. Total species richness, Shannon Index, and Saprobity index $S$ (Eq. 1) in the sampling points of the Alakol Lake with geographical coordinates in 2015-2017

\begin{tabular}{c|c|c|c|c|c}
\hline Area & North & East & Shannon Index & No of Species & $\begin{array}{c}\text { Saprobity index } \\
\text { S }\end{array}$ \\
\hline Kok & 45.52 .23 .30 & 81.28 .59 .48 & 3.43 & 33 & 1.63 \\
Kok & 45.52 .11 .20 & 81.39 .06 .06 & 2.45 & 12 & 1.87 \\
Kok & 45.52 .11 .20 & 81.39 .06 .06 & 3.50 & 36 & 1.48 \\
Kok & 45.51 .58 .47 & 81.39 .14 .38 & 1.55 & 5 & 2.22 \\
Kok & 45.51 .44 .20 & 81.39 .28 .04 & 2.58 & 14 & 1.19 \\
Kok & 45.52 .49 .98 & 81.34 .48 .81 & 2.58 & 14 & 1.26 \\
Kok & 45.52 .49 .98 & 81.34 .48 .81 & 1.08 & 3 & 1.17 \\
Kok & 45.52 .40 .77 & 81.38 .53 .59 & 1.59 & 6 & - \\
Aksh & 45.57 .41 .41 & 81.33 .25 .35 & 1.31 & 4 & 1.69 \\
Aksh & 45.57 .41 .41 & 81.33 .25 .35 & 1.33 & 4 & 1.56 \\
Aksh & 45.57 .03 .24 & 81.34 .43 .62 & 2.47 & 13 & 1.30 \\
Kam & 46.27 .16 .87 & 81.31 .07 .07 & 1.77 & 6 & 1.77 \\
Kam & 46.27 .22 .24 & 81.31 .43 .05 & 2.18 & 9 & 1.75 \\
Kam & 46.28 .56 .08 & 81.32 .44 .87 & 1.39 & 4 & 1.31 \\
Kam & 46.28 .11 .05 & 81.32 .42 .34 & 1.31 & 4 & 1.28 \\
Kam & 46.28 .16 .99 & 81.32 .45 .65 & 0.56 & 2 & 2.13 \\
Kam & 46.28 .27 .84 & 81.32 .32 .00 & 2.24 & 10 & 2.16 \\
Kam & 46.28 .27 .84 & 81.32 .32 .00 & 0.00 & 1 & 2.00 \\
Kam & 46.28 .27 .09 & 81.32 .04 .02 & 1.10 & 3 & - \\
Kam & 46.28 .30 .24 & 81.31 .49 .37 & 2.86 & 19 & 1.82 \\
Kam & 46.28 .30 .24 & 81.31 .49 .37 & 1.88 & 8 & \\
\hline Abbrev & 10. Kok, Ko & 4.09 & \\
\hline
\end{tabular}

Abbreviation: Kok, Koktuma area; Aksh, Akshi area; Kam, Kamyskala area

Altogether 208 species and infraspecies of algae and cyanobacteria from five taxonomic Divisions were revealed from 21 samples of phytoplankton and microphytobenthos in the Alakol Lake (Jiyenbekovet al., 2018). We compared the list of species of the Alakol Lake with our database (Barinova et al., 2006) and find that all 208 taxa can be indicators of environmental properties of the lake water that represent of $100 \%$ of revealed diversity list. So, Table 2 are represented ecological parameters of algae-indicator species of the Alakol Lake. 
Table 2. Ecological properties of indicator taxa of algae of the Alakol Lake in 2015-2017 according Barinova et al., 2006

\begin{tabular}{|c|c|c|c|c|c|c|c|c|c|c|c|}
\hline Taxa & Hab & $\mathbf{T}$ & $\mathbf{O x y}$ & pH & pH-range & Sal & D & Sap & $\mathbf{S}$ & Tro & $\begin{array}{l}\text { Aut- } \\
\text { Het }\end{array}$ \\
\hline \multicolumn{12}{|l|}{ Cyanobacteria } \\
\hline $\begin{array}{c}\text { Anabaena cylindrica } \\
\text { Lemmermann }\end{array}$ & P-B, S & - & aer & - & - & - & - & $b-o$ & 1.70 & - & - \\
\hline $\begin{array}{c}\text { Anabaena oscillarioides } \\
\text { Bory ex Bornet \& Flahault }\end{array}$ & P-B & - & - & - & - & - & - & $b$ & 2.00 & - & - \\
\hline $\begin{array}{c}\text { Arthrospira jenneri } \\
\text { Stizenberger ex Gomont }\end{array}$ & P-B & - & st & - & $4.7-9.0$ & - & - & $b-p$ & 3.70 & $\mathrm{~m}$ & - \\
\hline $\begin{array}{l}\text { Chroococcus minutus } \\
\text { (Kützing) Nägeli }\end{array}$ & P-B & - & - & ind & - & $\mathrm{i}$ & - & o-a & 1.80 & o-m & - \\
\hline $\begin{array}{c}\text { Chroococcus tenax } \\
\text { (Kirchner) Hieronymus }\end{array}$ & $\mathrm{B}, \mathrm{S}$ & - & aer & - & - & - & - & - & - & o & - \\
\hline $\begin{array}{l}\text { Chroococcus turgidus } \\
\text { (Kützing) Nägeli }\end{array}$ & P-B, S & - & aer & alf & - & hl & - & $x-b$ & 0.80 & - & - \\
\hline $\begin{array}{c}\text { Gloeocapsa turgida } \mathrm{f} . \\
\text { subnuda (Hansgirg) } \\
\text { Hollerbach }\end{array}$ & P-B, S & - & aer & alf & - & hl & - & $x-b$ & 0.80 & - & - \\
\hline $\begin{array}{c}\text { Gloeocapsa violacea } \\
\text { Kützing }\end{array}$ & $\mathrm{S}$ & - & - & - & - & - & - & - & - & - & - \\
\hline $\begin{array}{l}\text { Gloeotrichia intermedia } \\
\text { (Lemmermann) Geitler }\end{array}$ & B,Ep & - & - & - & - & - & - & - & - & o & - \\
\hline $\begin{array}{l}\text { Merismopedia glauca } \\
\text { (Ehrenberg) Kützing }\end{array}$ & P-B & - & - & ind & - & $\mathrm{i}$ & - & b-o & 1.75 & o-m & - \\
\hline $\begin{array}{c}\text { Merismopedia punctata } \\
\text { Meyen }\end{array}$ & P-B & - & - & ind & - & $\mathrm{i}$ & - & $\mathrm{o}-\mathrm{a}$ & 1.80 & me & - \\
\hline $\begin{array}{c}\text { Nodularia harveyana } \\
\text { Thuret ex Bornet \& } \\
\text { Flahault }\end{array}$ & $\mathrm{B}, \mathrm{S}$ & - & - & - & - & $\mathrm{mh}$ & - & $\mathrm{o}$ & 1.20 & - & - \\
\hline $\begin{array}{l}\text { Nodularia spumigena } \\
\text { Mertens ex Bornet \& }\end{array}$ & $\mathrm{B}, \mathrm{S}$ & - & - & - & - & - & - & $\mathrm{o}-\mathrm{a}$ & 1.80 & - & - \\
\hline
\end{tabular}




\begin{tabular}{|c|c|c|c|c|c|c|c|c|c|c|c|}
\hline Taxa & Hab & $\mathbf{T}$ & Oxy & pH & pH-range & Sal & D & Sap & $\mathbf{S}$ & Tro & $\begin{array}{l}\text { Aut- } \\
\text { Het }\end{array}$ \\
\hline \multicolumn{12}{|l|}{ Flahault } \\
\hline $\begin{array}{c}\text { Nostoc linckia } \text { Bornet ex } \\
\text { Bornet \& Flahault }\end{array}$ & P-B, Ep & - & - & - & - & - & - & b-o & 1.60 & - & - \\
\hline $\begin{array}{c}\text { Nostoc zetterstedtii } \\
\text { Areschoug ex Bornet \& } \\
\text { Flahault }\end{array}$ & B & - & - & - & - & - & - & - & - & - & - \\
\hline $\begin{array}{l}\text { Oscillatoria princeps } \\
\text { Vaucher ex Gomont }\end{array}$ & P-B, S & - & st-str & - & - & - & - & $\mathrm{a}-\mathrm{o}$ & 2.80 & $\mathrm{o}-\mathrm{m}$ & - \\
\hline $\begin{array}{l}\text { Oscillatoria sancta } \\
\text { Kützing ex Gomont }\end{array}$ & P-B, S & eterm & $\begin{array}{l}\text { st-str, } \\
\text { aer }\end{array}$ & - & - & $\mathrm{i}$ & - & $\mathrm{a}-\mathrm{o}$ & 2.70 & me & - \\
\hline $\begin{array}{c}\text { Spirulina labyrinthiformis } \\
\text { Gomont }\end{array}$ & P-B & - & st-str & - & - & - & - & - & - & - & - \\
\hline $\begin{array}{c}\text { Spirulina major Kützing ex } \\
\text { Gomont }\end{array}$ & P-B, S & warm & st & - & - & $\mathrm{hl}$ & - & $\mathrm{a}$ & 3.40 & - & - \\
\hline $\begin{array}{c}\text { Spirulina subsalsa Oersted } \\
\text { ex Gomont }\end{array}$ & B & - & st-str & - & - & - & - & $\mathrm{a}-\mathrm{o}$ & 2.70 & - & - \\
\hline $\begin{array}{c}\text { Trichodesmium lacustre } \\
\text { Klebahn }\end{array}$ & $\mathrm{P}$ & - & st & - & - & - & - & - & - & - & - \\
\hline $\begin{array}{c}\text { Trichormus variabilis } \\
\text { (Kützing ex Bornet \& } \\
\text { Flahault) Komárek \& } \\
\text { Anagnostidis } \\
\end{array}$ & P-B, S & - & st & - & - & $\mathrm{mh}$ & - & - & - & - & - \\
\hline
\end{tabular}

\section{Bacillariophyta}

\begin{tabular}{|c|c|c|c|c|c|c|c|c|c|c|c|}
\hline Amphora eximia J.R.Carter & $\mathrm{B}$ & - & - & alf & 7.7 & $\mathrm{hb}$ & sx & $\mathrm{O}$ & 1.00 & - & - \\
\hline $\begin{array}{c}\text { Amphora lineolata } \\
\text { Ehrenberg }\end{array}$ & B & - & - & - & - & hl & - & - & - & - & - \\
\hline $\begin{array}{c}\text { Amphora ovalis (Kützing) } \\
\text { Kützing }\end{array}$ & $\mathrm{B}$ & temp & st-str & alf & $6.2-9.0$ & $\mathrm{i}$ & sx & $o-b$ & 1.50 & me & ate \\
\hline $\begin{array}{l}\text { Anomoeoneis costata } \\
\text { (Kützing) Hustedt }\end{array}$ & $\mathrm{B}$ & - & - & - & - & $\mathrm{mh}$ & - & $\mathrm{a}-\mathrm{O}$ & 2.70 & - & - \\
\hline
\end{tabular}




\begin{tabular}{|c|c|c|c|c|c|c|c|c|c|c|c|}
\hline Taxa & Hab & $\mathbf{T}$ & Oxy & pH & pH-range & Sal & D & Sap & $\mathbf{S}$ & Tro & $\begin{array}{l}\text { Aut- } \\
\text { Het }\end{array}$ \\
\hline $\begin{array}{c}\text { Anomoeoneis } \\
\text { sphaerophora } \text { Pfitzer }\end{array}$ & B & warm & st-str & alf & $6.3-9.0$ & hl & - & $a-o$ & 2.70 & me & ate \\
\hline $\begin{array}{l}\text { Aulacoseira ambigua } \\
\text { (Grunow) Simonsen }\end{array}$ & $\mathrm{P}$ & - & st-str & alf & $6.0-8.5$ & $\mathrm{i}$ & $\mathrm{sp}$ & b-o & 1.70 & o-m & ate \\
\hline $\begin{array}{c}\text { Brebissonia lanceolata } \\
\text { (C.Agardh) R.K.Mahoney } \\
\text { \& Reimer }\end{array}$ & P-B & temp & - & alf & $4.8-9.0$ & $\mathrm{i}$ & - & $\mathrm{X}-\mathrm{O}$ & 0.40 & - & - \\
\hline $\begin{array}{c}\text { Caloneis amphisbaena } \\
\text { (Bory) Cleve }\end{array}$ & B & - & st-str & alf & - & $\mathrm{i}$ & - & $\mathrm{b}$ & 2.30 & me & ate \\
\hline $\begin{array}{l}\text { Caloneis amphisbaena var. } \\
\text { subsalina (Donkin) Cleve }\end{array}$ & B & - & st-str & alf & - & hl & - & $\mathrm{b}$ & 2.30 & $\mathrm{e}$ & ate \\
\hline $\begin{array}{c}\text { Caloneis latiuscula } \\
\text { (Kützing) Cleve }\end{array}$ & B & - & st & ind & - & $\mathrm{i}$ & - & o & 1.00 & - & - \\
\hline $\begin{array}{c}\text { Caloneis molaris (Grunow) } \\
\text { Krammer }\end{array}$ & B & - & str & ind & - & $\mathrm{i}$ & es & o & 1.00 & ot & - \\
\hline $\begin{array}{c}\text { Caloneis silicula } \\
\text { (Ehrenberg) Cleve }\end{array}$ & B & - & st & ind & $6.3-9.0$ & $\mathrm{i}$ & $\mathrm{sp}$ & o & 1.30 & o-m & ats \\
\hline $\begin{array}{c}\text { Caloneis westii (W.Smith) } \\
\text { Hendey }\end{array}$ & B & - & - & - & - & hl & - & - & - & - & - \\
\hline $\begin{array}{c}\text { Cavinula pusio (Cleve) } \\
\text { Lange-Bertalot }\end{array}$ & B & - & - & ind & - & $\mathrm{i}$ & - & o & 1.00 & - & - \\
\hline $\begin{array}{c}\text { Cocconeis placentula var. } \\
\text { euglypta (Ehrenberg) } \\
\text { Grunow }\end{array}$ & P-B & temp & st-str & alf & $5.5-9$ & $\mathrm{i}$ & SX & o & 1.30 & o-m & ate \\
\hline $\begin{array}{c}\text { Cosmioneis pusilla } \\
\text { (W.Smith) D.G.Mann \& } \\
\text { A.J.Stickle }\end{array}$ & P-B, aer & - & str & ind & - & hl & $\mathrm{sp}$ & $\mathrm{o}-\mathrm{a}$ & 1.80 & $\mathrm{o}-\mathrm{m}$ & ats \\
\hline $\begin{array}{c}\text { Craticula ambigua } \\
\text { (Ehrenberg) D.G.Mann in } \\
\text { Round, R.M.Crawford \& } \\
\text { D.G.Mann }\end{array}$ & B & warm & st & alf & $5.5-6.0$ & $\mathrm{i}$ & es & $\mathrm{b}$ & 2.30 & me & - \\
\hline
\end{tabular}

APPLIED ECOLOGY AND ENVIRONMENTAL RESEARCH 16(6):7799-7831.

http://www.aloki.hu • ISSN 15891623 (Print) • ISSN 17850037 (Online)

DOI: http://dx.doi.org/10.15666/aeer/1606_77997831

( 2018 , ALÖKI Kft., Budapest, Hungary 


\begin{tabular}{|c|c|c|c|c|c|c|c|c|c|c|c|}
\hline Taxa & Hab & $\mathbf{T}$ & $\mathbf{O x y}$ & pH & pH-range & Sal & $\mathbf{D}$ & Sap & $\mathbf{S}$ & Tro & $\begin{array}{l}\text { Aut- } \\
\text { Het }\end{array}$ \\
\hline $\begin{array}{l}\text { Ctenophora pulchella } \\
\text { (Ralfs ex Kützing) } \\
\text { D.M.Williams \& Round }\end{array}$ & P-B & - & st-str & alf & - & $\mathrm{i}$ & - & $b$ & 2.30 & $\mathrm{o}-\mathrm{m}$ & ate \\
\hline $\begin{array}{c}\text { Ctenophora pulchella var. } \\
\text { lacerata (Hustedt) } \\
\text { Bukhtiyarova }\end{array}$ & $\mathrm{B}$ & - & - & - & - & $\mathrm{mh}$ & - & - & - & - & - \\
\hline $\begin{array}{c}\text { Cyclotella meneghiniana } \\
\text { Kützing }\end{array}$ & P-B & temp & st & alf & $5.5-9.0$ & hl & $\mathrm{sp}$ & $\mathrm{a}-\mathrm{o}$ & 2.80 & e & hne \\
\hline $\begin{array}{c}\text { Cyclotella pseudostelligera } \\
\text { Hustedt }\end{array}$ & $\mathrm{P}$ & - & st-str & ind & - & $\mathrm{i}$ & - & $b$ & 2.30 & $\mathrm{e}$ & ate \\
\hline $\begin{array}{c}\text { Cylindrotheca closterium } \\
\text { (Ehrenberg) Reimann \& } \\
\text { J.C.Lewin }\end{array}$ & B & - & - & alf & - & $\mathrm{i}$ & - & $\mathrm{b}$ & 2.00 & - & - \\
\hline Cymbella affinis Kützing & B & temp & st-str & alf & - & $\mathrm{i}$ & sx & o & 1.10 & ot & ats \\
\hline $\begin{array}{l}\text { Cymbella affinis var. } \\
\text { neoprocera } \text { W.Silva }\end{array}$ & B & temp & st-str & alf & - & $\mathrm{i}$ & sX & o & 1.10 & ot & ats \\
\hline $\begin{array}{c}\text { Cymbella aspera } \\
\text { (Ehrenberg) Cleve }\end{array}$ & $\mathrm{B}$ & - & st-str & neu & - & $\mathrm{i}$ & es & $\mathrm{x}$ & 0.30 & o-e & ats \\
\hline $\begin{array}{c}\text { Cymbella cistula } \\
\text { (Ehrenberg) O.Kirchner }\end{array}$ & B & - & st-str & alf & 8.0 & $\mathrm{i}$ & sx & o & 1.20 & e & ats \\
\hline $\begin{array}{c}\text { Cymbella cymbiformis } \\
\text { C.Agardh }\end{array}$ & B & temp & str & ind & $6.2-9$ & $\mathrm{i}$ & sx & $b$ & 2.00 & o-m & ats \\
\hline Cymbella excisa Kützing & $\mathrm{B}$ & - & - & - & - & - & - & - & - & - & - \\
\hline $\begin{array}{c}\text { Cymbella helvetica } \\
\text { Kützing }\end{array}$ & B & - & str & ind & - & $\mathrm{i}$ & - & $\mathrm{O}-\mathrm{X}$ & 0.60 & o-m & - \\
\hline $\begin{array}{c}\text { Cymbella parva (W.Smith) } \\
\text { Kirchner }\end{array}$ & $\mathrm{B}$ & - & - & ind & - & $\mathrm{i}$ & - & $b$ & 2.00 & o-m & - \\
\hline $\begin{array}{c}\text { Cymbella pseudoaffinis } \\
\text { Tynni }\end{array}$ & - & - & - & - & - & - & - & - & - & - & - \\
\hline Cymbella simonsenii & $\mathrm{B}$ & - & - & - & - & - & - & $\mathrm{O}$ & 1.00 & - & - \\
\hline
\end{tabular}




\begin{tabular}{|c|c|c|c|c|c|c|c|c|c|c|c|}
\hline Taxa & Hab & $\mathbf{T}$ & Oxy & pH & pH-range & Sal & $\mathbf{D}$ & Sap & $\mathbf{S}$ & Tro & $\begin{array}{l}\text { Aut- } \\
\text { Het }\end{array}$ \\
\hline \multicolumn{12}{|l|}{ Krammer } \\
\hline $\begin{array}{c}\text { Cymbella tumida } \\
\text { (Brébisson) Van Heurck }\end{array}$ & B & temp & str & alf & $6.8-9$ & $\mathrm{i}$ & SX & $\mathrm{b}$ & 2.20 & me & ats \\
\hline $\begin{array}{l}\text { Cymbopleura inaequalis } \\
\text { (Ehrenberg) Krammer }\end{array}$ & B & - & st-str & ind & - & $\mathrm{i}$ & - & o & 1.10 & $\mathrm{~m}$ & ats \\
\hline $\begin{array}{c}\text { Diatoma ehrenbergii } \\
\text { Kützing }\end{array}$ & B & - & st-str & alf & - & I & - & $\mathrm{o}-\mathrm{X}$ & 0.70 & o-m & ate \\
\hline $\begin{array}{l}\text { Diatoma moniliformis } \\
\text { (Kützing) D.M.Williams }\end{array}$ & P-B & - & st-str & alf & - & $\mathrm{i}$ & - & o & 1.30 & o-m & - \\
\hline $\begin{array}{c}\text { Diatoma vulgaris var. } \\
\text { brevis Grunow }\end{array}$ & P-B & - & st-str & alb & - & $\mathrm{i}$ & SX & $\mathrm{b}$ & 2.20 & me & ate \\
\hline $\begin{array}{c}\text { Encyonema cespitosum } \\
\text { Kützing }\end{array}$ & B & - & - & - & - & $\mathrm{i}$ & $\mathrm{SX}$ & o & 1.30 & o-e & - \\
\hline $\begin{array}{c}\text { Encyonema leibleinii } \\
\text { (C.Agardh) W.J.Silva, } \\
\text { R.Jahn, T.A.Veiga Ludwig } \\
\text { \& M.Menezes }\end{array}$ & P-B & - & str & alb & $4.7-9.0$ & $\mathrm{i}$ & es & o & 1.30 & $\mathrm{e}$ & ats \\
\hline $\begin{array}{l}\text { Encyonema minutum } \\
\text { (Hilse) D.G.Mann }\end{array}$ & B & - & st-str & ind & 6.2 & $\mathrm{i}$ & SX & o & 1.20 & o-e & ate \\
\hline $\begin{array}{l}\text { Encyonema obscurum } \\
\text { (Krasske) D.G.Mann }\end{array}$ & B & - & - & ind & - & - & - & o & 1.00 & ot & - \\
\hline $\begin{array}{c}\text { Encyonema silesiacum } \\
\text { (Bleisch) D.G.Mann in } \\
\text { Round, R.M.Crawford \& } \\
\text { D.G.Mann } \\
\end{array}$ & B & - & st-str & ind & $6.2-7.7$ & $\mathrm{i}$ & Sx & o & 1.20 & o-e & ate \\
\hline $\begin{array}{c}\text { Encyonema } \\
\text { subventricosum } \\
\text { (Cholnoky) Krammer }\end{array}$ & B & - & st-str & ind & $6.2-7.9$ & $\mathrm{i}$ & SX & o & 1.20 & o-e & ate \\
\hline $\begin{array}{l}\text { Entomoneis paludosa } \\
\text { (W.Smith) Reimer in }\end{array}$ & P-B & - & - & alf & - & hl & - & $\mathrm{b}-\mathrm{a}$ & 2.50 & $\mathrm{~m}$ & - \\
\hline
\end{tabular}




\begin{tabular}{|c|c|c|c|c|c|c|c|c|c|c|c|}
\hline Taxa & Hab & $\mathbf{T}$ & Oxy & pH & pH-range & Sal & D & Sap & $\mathbf{S}$ & Tro & $\begin{array}{l}\text { Aut- } \\
\text { Het }\end{array}$ \\
\hline \multicolumn{12}{|l|}{ R.M.Patrick \& Reimer } \\
\hline $\begin{array}{c}\text { Entomoneis paludosa var. } \\
\text { subsalina (Cleve) } \\
\text { Krammer in Lange- } \\
\text { Bertalot \& Krammer } \\
\end{array}$ & B & - & - & - & - & hl & - & - & - & - & - \\
\hline $\begin{array}{c}\text { Epithemia adnata } \text { var. } \\
\text { porcellus (Kützing) R.Ross }\end{array}$ & B & - & - & alf & - & $\mathrm{i}$ & - & $\mathrm{b}$ & 2.00 & me & - \\
\hline $\begin{array}{c}\text { Epithemia adnata var. } \\
\text { saxonica (Kützing) } \\
\text { R.M.Patrick in Patrick \& } \\
\text { Reimer }\end{array}$ & B & temp & - & alf & $4.8-9.0$ & $\mathrm{i}$ & - & o & 1.20 & me & - \\
\hline $\begin{array}{c}\text { Epithemia argus var. } \\
\text { alpestris (W.Smith) } \\
\text { Grunow }\end{array}$ & B & - & - & ind & - & $\mathrm{i}$ & - & $\mathrm{O}-\mathrm{X}$ & 0.70 & $\mathrm{~m}$ & - \\
\hline $\begin{array}{l}\text { Epithemia argus var. } \\
\text { angustata Tarnavschi }\end{array}$ & B & - & - & ind & - & $\mathrm{i}$ & - & - & - & - & - \\
\hline $\begin{array}{c}\text { Epithemia gibba } \\
\text { (Ehrenberg) Kützing }\end{array}$ & B & temp & - & alb & $6.2-9$ & $\mathrm{i}$ & es & $\mathrm{X}-\mathrm{O}$ & 0.40 & - & - \\
\hline Epithemia sorex Kützing & B & temp & st-str & alf & $5.0-9.0$ & $\mathrm{i}$ & sx & o & 1.10 & me & ats \\
\hline $\begin{array}{c}\text { Epithemia turgida } \\
\text { (Ehrenberg) Kützing }\end{array}$ & B & temp & st & alf & $5.0-9.0$ & $\mathrm{i}$ & sx & $x-b$ & 0.90 & me & ats \\
\hline $\begin{array}{c}\text { Eunotia flexuosa } \\
\text { (Brébisson ex Kützing) } \\
\text { Kützing } \\
\end{array}$ & B & - & st-str & acf & $4.5-7.2$ & $\mathrm{i}$ & - & $x-b$ & 0.80 & o-m & ats \\
\hline $\begin{array}{c}\text { Fragilaria acus (Kützing) } \\
\text { Lange-Bertalot in } \\
\text { Krammer \& Lange- } \\
\text { Bertalot }\end{array}$ & $\mathrm{P}$ & - & st-str & alb & - & $\mathrm{i}$ & es & o-a & 1.80 & - & - \\
\hline
\end{tabular}




\begin{tabular}{|c|c|c|c|c|c|c|c|c|c|c|c|}
\hline Taxa & Hab & $\mathbf{T}$ & $\mathbf{O x y}$ & pH & pH-range & Sal & D & Sap & $\mathbf{S}$ & Tro & $\begin{array}{l}\text { Aut- } \\
\text { Het }\end{array}$ \\
\hline $\begin{array}{l}\text { Fragilaria alpestris } \\
\text { Krasske ex Hustedt }\end{array}$ & P-B, aer & - & - & ind & - & $\mathrm{i}$ & - & $\mathrm{o}$ & 1.00 & ot & - \\
\hline Fragilaria bidens Heiberg & P-B & - & str & alf & - & $\mathrm{i}$ & - & $\mathrm{b}$ & 2.00 & $\mathrm{e}$ & ats \\
\hline $\begin{array}{c}\text { Fragilaria capucina } \\
\text { Desmazières } \\
\end{array}$ & P-B & - & - & ind & 7.7 & $\mathrm{i}$ & es & $\mathrm{b}-\mathrm{o}$ & 1.60 & $\mathrm{~m}$ & - \\
\hline $\begin{array}{c}\text { Fragilaria crotonensis } \\
\text { Kitton }\end{array}$ & $\mathrm{P}$ & - & st-str & alf & 7.5 & I & es & $o-b$ & 1.50 & $\mathrm{~m}$ & ate \\
\hline $\begin{array}{c}\text { Fragilaria rumpens } \\
\text { (Kützing) G.W.F.Carlson }\end{array}$ & B & - & - & alf & 7.7 & $\mathrm{i}$ & es & $\mathrm{x}-\mathrm{a}$ & 1.55 & - & - \\
\hline $\begin{array}{l}\text { Fragilaria tenera } \\
\text { (W.Smith) Lange-Bertalot }\end{array}$ & P-B & - & str & acf & - & $\mathrm{hb}$ & SX & $\mathrm{b}$ & 2.30 & $\mathrm{o}-\mathrm{m}$ & ats \\
\hline $\begin{array}{c}\text { Fragilaria tenera } \text { var. } \\
\text { nanana (Lange-Bertalot) } \\
\text { Lange-Bertalot \& S.Ulrich }\end{array}$ & P-B & - & str & ind & - & $\mathrm{hb}$ & - & o & 1.00 & me & ats \\
\hline $\begin{array}{c}\text { Frustulia crassinervia } \\
\text { (Brébisson ex W.Smith) } \\
\text { Lange-Bertalot \& } \\
\text { Krammer in Lange- } \\
\text { Bertalot \& Metzeltin } \\
\end{array}$ & B & - & str & acf & 6.4 & $\mathrm{hb}$ & es & $\mathrm{X}-\mathrm{O}$ & 0.50 & ot & ats \\
\hline $\begin{array}{l}\text { Frustulia rhomboides } \\
\text { (Ehrenberg) De Toni }\end{array}$ & B & - & st & acf & 5.5 & $\mathrm{hb}$ & es & $\mathrm{x}$ & 0.30 & ot & ats \\
\hline $\begin{array}{l}\text { Gomphoneis clevei } \\
\text { (Fricke) Gil }\end{array}$ & B & - & - & alf & - & $\mathrm{i}$ & - & $\mathrm{x}$ & 0.30 & ot & - \\
\hline $\begin{array}{c}\text { Gomphonema angustatum } \\
\text { (Kützing) Rabenhorst }\end{array}$ & B & - & st-str & ind & 6.6 & $\mathrm{i}$ & es & o & 1.30 & $\mathrm{o}-\mathrm{m}$ & - \\
\hline $\begin{array}{c}\text { Gomphonema calcareum } \\
\text { Cleve }\end{array}$ & B & - & st-str & alf & - & $\mathrm{i}$ & - & $\mathrm{b}$ & 2.30 & $\mathrm{o}-\mathrm{m}$ & ate \\
\hline $\begin{array}{c}\text { Gomphonema constrictum } \\
\text { Ehrenberg }\end{array}$ & B & - & - & alf & - & $\mathrm{i}$ & es & $o-b$ & 1.50 & $\mathrm{e}$ & - \\
\hline
\end{tabular}




\begin{tabular}{|c|c|c|c|c|c|c|c|c|c|c|c|}
\hline Taxa & Hab & $\mathbf{T}$ & Oxy & pH & pH-range & Sal & D & Sap & $\mathbf{S}$ & Tro & $\begin{array}{l}\text { Aut } \\
\text { Het }\end{array}$ \\
\hline $\begin{array}{c}\text { Gomphonema gracile } \\
\text { Ehrenberg }\end{array}$ & B & temp & st & alf & $5-9$ & $\mathrm{i}$ & es & $\mathrm{x}-\mathrm{b}$ & 0.80 & $\mathrm{~m}$ & ats \\
\hline $\begin{array}{c}\text { Gomphonema insigne } \\
\text { W.Gregory }\end{array}$ & B & - & - & - & - & $\mathrm{i}$ & - & - & - & - & - \\
\hline $\begin{array}{c}\text { Gomphonema longiceps } \\
\text { var. subclavatum Grunow }\end{array}$ & B & - & str & ind & - & $\mathrm{i}$ & es & o-b & 1.40 & - & - \\
\hline $\begin{array}{l}\text { Gomphonema minutum } \\
\text { (C.Agardh) C.Agardh }\end{array}$ & B & - & - & alf & - & $\mathrm{i}$ & es & o-b & 1.50 & $\mathrm{e}$ & - \\
\hline $\begin{array}{l}\text { Gomphonema olivaceum } \\
\text { (Hornemann) Brébisson }\end{array}$ & B & - & st-str & alf & $7.5-8.0$ & $\mathrm{i}$ & es & o-b & 1.45 & e & ate \\
\hline $\begin{array}{l}\text { Gomphonema olivaceum } \\
\text { var. minutissimum Hustedt }\end{array}$ & B & - & str & alf & - & $\mathrm{i}$ & - & o & 1.20 & o-m & ats \\
\hline $\begin{array}{l}\text { Gomphonema parvulum } \\
\text { (Kützing) Kützing }\end{array}$ & B & temp & str & ind & 4.5 & $\mathrm{i}$ & es & $\mathrm{b}$ & 2.35 & o-m & hne \\
\hline $\begin{array}{l}\text { Gomphonema truncatum } \\
\text { Ehrenberg }\end{array}$ & B & - & st-str & ind & - & $\mathrm{i}$ & es & o-b & 1.40 & me & ats \\
\hline $\begin{array}{c}\text { Gomphonema ventricosum } \\
\text { W.Gregory }\end{array}$ & B & cool & str & ind & - & $\mathrm{i}$ & - & $\mathrm{x}$ & 0.30 & ot & ats \\
\hline $\begin{array}{l}\text { Gomphonema vibrio } \\
\text { Ehrenberg }\end{array}$ & B & - & st-str & ind & - & $\mathrm{i}$ & es & o & 1.10 & - & - \\
\hline $\begin{array}{l}\text { Gyrosigma acuminatum } \\
\text { (Kützing) Rabenhorst }\end{array}$ & B & cool & st-str & alf & - & $\mathrm{i}$ & es & o-a & 1.95 & me & ate \\
\hline $\begin{array}{l}\text { Gyrosigma scalproides } \\
\text { (Rabenhorst) Cleve }\end{array}$ & B & - & - & alf & - & $\mathrm{i}$ & es & $\mathrm{b}$ & 2.20 & - & - \\
\hline $\begin{array}{l}\text { Halamphora coffeiformis } \\
\text { (C.Agardh) Levkov }\end{array}$ & B & - & st-str & alf & - & $\mathrm{mh}$ & - & a & 3.00 & e & ate \\
\hline $\begin{array}{l}\text { Halamphora normanii } \\
\text { (Rabenhorst) Levkov }\end{array}$ & B & - & aer & alf & - & $\mathrm{hb}$ & - & $\mathrm{x}$ & 0.10 & $\mathrm{~m}$ & ats \\
\hline
\end{tabular}




\begin{tabular}{|c|c|c|c|c|c|c|c|c|c|c|c|}
\hline Taxa & Hab & $\mathbf{T}$ & $\mathbf{O x y}$ & pH & pH-range & Sal & D & Sap & $\mathbf{S}$ & Tro & $\begin{array}{l}\text { Aut- } \\
\text { Het }\end{array}$ \\
\hline $\begin{array}{l}\text { Halamphora veneta } \\
\text { (Kützing) Levkov }\end{array}$ & B & - & st-str & alf & - & $\mathrm{i}$ & es & a-o & 2.60 & $\mathrm{e}$ & ate \\
\hline $\begin{array}{c}\text { Hannaea arcus } \\
\text { (Ehrenberg) R.M.Patrick in } \\
\text { R.M.Patrick \& } \\
\text { C.W.Reimer }\end{array}$ & B & temp & str & alf & $5.5-7.5$ & $\mathrm{i}$ & es & $\mathrm{x}$ & 0.30 & $\mathrm{o}-\mathrm{m}$ & ats \\
\hline $\begin{array}{c}\text { Hannaea arcus var. } \\
\text { amphioxys (Rabenhorst) } \\
\text { R.M.Patrick }\end{array}$ & B & cool & str & alf & - & $\mathrm{i}$ & - & $\mathrm{X}$ & 0.30 & - & - \\
\hline $\begin{array}{c}\text { Hannaea inaequidentata } \\
\text { (Lagerstedt) S.I.Genkal \& } \\
\text { V.G.Kharitonov }\end{array}$ & P-B & - & - & neu & - & $\mathrm{hb}$ & - & o & 1.00 & ot & - \\
\hline $\begin{array}{c}\text { Hantzschia amphioxys } \\
\text { (Ehrenberg) Grunow in } \\
\text { Cleve \& Grunow }\end{array}$ & B & temp & st-str & ind & - & I & es & $\mathrm{o}-\mathrm{a}$ & 1.90 & o-e & ate \\
\hline $\begin{array}{c}\text { Hantzschia amphioxys var. } \\
\text { constricta Pantocsek }\end{array}$ & $\mathrm{B}$ & - & - & ind & - & $\mathrm{i}$ & - & - & - & - & - \\
\hline $\begin{array}{c}\text { Mastogloia albertii } \\
\text { A.Pavlov, E.Jovanovska, } \\
\text { C.E.Wetzel, L.Ector \& } \\
\text { Z.Levkov }\end{array}$ & $\mathrm{B}$ & - & - & alf & - & hl & - & - & - & - & - \\
\hline $\begin{array}{c}\text { Mastogloia baltica } \\
\text { Grunow }\end{array}$ & $\mathrm{B}$ & - & - & - & - & $\mathrm{mh}$ & - & - & - & - & - \\
\hline $\begin{array}{c}\text { Mastogloia grevillei } \\
\text { W.Smith }\end{array}$ & B & - & - & alf & - & $\mathrm{hl}$ & - & b & 2.00 & e & - \\
\hline $\begin{array}{l}\text { Mastogloia pumila } \\
\text { (Grunow) Cleve }\end{array}$ & B & - & - & - & - & $\mathrm{mh}$ & - & - & - & - & - \\
\hline $\begin{array}{c}\text { Mastogloia smithii } \\
\text { Thwaites ex W.Smith }\end{array}$ & B & - & - & alf & - & $\mathrm{mh}$ & SX & o & 1.30 & me & - \\
\hline Navicula cuspidata f. & $\mathrm{B}$ & - & st & - & - & $\mathrm{i}$ & - & $\mathrm{b}$ & 2.00 & - & - \\
\hline
\end{tabular}

APPLIED ECOLOGY AND ENVIRONMENTAL RESEARCH 16(6):7799-7831.

http://www.aloki.hu • ISSN 15891623 (Print) • ISSN 17850037 (Online)

DOI: http://dx.doi.org/10.15666/aeer/1606_77997831

( 2018 , ALÖKI Kft., Budapest, Hungary 


\begin{tabular}{|c|c|c|c|c|c|c|c|c|c|c|c|}
\hline Taxa & Hab & $\mathbf{T}$ & Oxy & $\mathbf{p H}$ & pH-range & Sal & D & Sap & $\mathbf{S}$ & Tro & $\begin{array}{l}\text { Aut- } \\
\text { Het }\end{array}$ \\
\hline \multicolumn{12}{|l|}{ primigena Dippel } \\
\hline $\begin{array}{c}\text { Navicula dicephala } \\
\text { Ehrenberg }\end{array}$ & B & - & - & ind & - & $\mathrm{i}$ & - & $\mathrm{o}-\mathrm{b}$ & 1.40 & - & - \\
\hline $\begin{array}{c}\text { Navicula pusilla var. } \\
\text { jacutica Kisselev }\end{array}$ & $\mathrm{B}$ & - & - & ind & - & hl & - & - & - & - & - \\
\hline Navicula radiosa Kützing & $\mathrm{B}$ & temp & st-str & ind & $5-9$ & $\mathrm{i}$ & es & o & 1.30 & me & ate \\
\hline $\begin{array}{c}\text { Navicula rhynchotella } \\
\text { Lange-Bertalot }\end{array}$ & B & - & - & alf & - & hl & es & $\mathrm{b}-\mathrm{a}$ & 2.55 & - & - \\
\hline $\begin{array}{c}\text { Navicula tenelloides } \\
\text { Hustedt }\end{array}$ & $\mathrm{B}, \mathrm{S}$ & - & str & alf & - & $\mathrm{i}$ & es & o & 1.00 & e & ats \\
\hline $\begin{array}{c}\text { Navicula tripunctata } \\
\text { (O.F.Müller) Bory in Bory } \\
\text { de Saint-Vincent }\end{array}$ & P-B & - & st-str & ind & - & $\mathrm{i}$ & es & $\mathrm{b}-\mathrm{o}$ & 1.70 & e & ate \\
\hline $\begin{array}{c}\text { Navicula trivialis Lange- } \\
\text { Bertalot }\end{array}$ & B & - & st-str & alf & 8.1 & $\mathrm{i}$ & $\mathrm{sp}$ & $\mathrm{b}-\mathrm{a}$ & 2.50 & $\mathrm{e}$ & ate \\
\hline $\begin{array}{l}\text { Neidiomorpha binodis } \\
\text { (Ehrenberg) M.Cantonati, } \\
\text { Lange-Bertalot \& N.Angeli }\end{array}$ & B & - & str & alf & - & $\mathrm{i}$ & - & o & 1.00 & me & ate \\
\hline $\begin{array}{c}\text { Neidium ampliatum } \\
\text { (Ehrenberg) Krammer in } \\
\text { Krammer \& Lange- } \\
\text { Bertalot }\end{array}$ & B & - & st & ind & - & I & es & $\mathrm{O}-\mathrm{X}$ & 0.60 & ot & - \\
\hline $\begin{array}{c}\text { Neidium apiculatum } \\
\text { Reimer }\end{array}$ & B & - & - & - & - & - & - & - & - & - & - \\
\hline $\begin{array}{l}\text { Neidium productum } \\
\text { (W.Smith) Cleve }\end{array}$ & P-B & temp & - & ind & - & $\mathrm{i}$ & sx & $\mathrm{x}-\mathrm{b}$ & 0.90 & ot & ats \\
\hline $\begin{array}{l}\text { Nitzschia acicularis } \\
\text { (Kützing) W.Smith }\end{array}$ & P-B & temp & - & alf & $7.85-8.15$ & $\mathrm{i}$ & es & a-o & 2.70 & $\mathrm{e}$ & hce \\
\hline
\end{tabular}

APPLIED ECOLOGY AND ENVIRONMENTAL RESEARCH 16(6):7799-7831.

http://www.aloki.hu • ISSN 15891623 (Print) • ISSN 17850037 (Online)

DOI: http://dx.doi.org/10.15666/aeer/1606_77997831

( 2018 , ALÖKI Kft., Budapest, Hungary 


\begin{tabular}{|c|c|c|c|c|c|c|c|c|c|c|c|}
\hline Taxa & Hab & $\mathbf{T}$ & Oxy & $\mathbf{p H}$ & pH-range & Sal & D & Sap & $\mathbf{S}$ & Tro & $\begin{array}{l}\text { Aut- } \\
\text { Het }\end{array}$ \\
\hline $\begin{array}{c}\text { Nitzschia filiformis } \\
\text { (W.Smith) Van Heurck }\end{array}$ & P-B & - & st-str & alf & - & hl & es & $\mathrm{b}-\mathrm{a}$ & 2.50 & $\mathrm{e}$ & hne \\
\hline $\begin{array}{c}\text { Nitzschia fonticola } \\
\text { (Grunow) Grunow in Van } \\
\text { Heurck }\end{array}$ & P-B & - & st-str & alf & $7.7-7.95$ & I & - & $\mathrm{o}-\mathrm{b}$ & 1.50 & me & ate \\
\hline $\begin{array}{c}\text { Nitzschia } \\
\text { gandersheimiensis f. } \\
\text { tenuirostris (Grunow) } \\
\text { Lange-Bertalot }\end{array}$ & B & - & - & ind & - & $\mathrm{i}$ & - & $\mathrm{a}-\mathrm{o}$ & 2.80 & - & - \\
\hline Nitzschia nana Grunow & - & - & str & ind & - & $\mathrm{i}$ & $\mathrm{sp}$ & $\mathrm{a}$ & 3.00 & $\mathrm{~m}$ & - \\
\hline Nitzschia obtusa W.Smith & $\mathrm{B}$ & - & - & - & - & hl & es & $\mathrm{b}-\mathrm{a}$ & 2.40 & $\mathrm{~m}$ & - \\
\hline $\begin{array}{c}\text { Nitzschia palea (Kützing) } \\
\text { W.Smith }\end{array}$ & P-B & temp & - & ind & $7-9$ & $\mathrm{i}$ & $\mathrm{sp}$ & $a-o$ & 2.80 & he & hce \\
\hline $\begin{array}{c}\text { Nitzschia palea var. } \\
\text { capitata Wislouch \& } \\
\text { Poretsky }\end{array}$ & B & - & - & ind & - & $\mathrm{i}$ & - & $\mathrm{b}$ & 2.00 & - & - \\
\hline $\begin{array}{c}\text { Nitzschia punctata var. } \\
\text { coarctata (Grunow) } \\
\text { Hustedt in A.W.F.Schimidt }\end{array}$ & B & - & - & - & - & - & - & - & - & - & - \\
\hline $\begin{array}{c}\text { Nitzschia scalpelliformis } \\
\text { Grunow in Cleve \& } \\
\text { Grunow }\end{array}$ & B & - & - & alf & - & hl & $\mathrm{sp}$ & $\mathrm{b}$ & 2.00 & me & - \\
\hline $\begin{array}{c}\text { Nitzschia sigma (Kützing) } \\
\text { W.Smith }\end{array}$ & B & temp & st-str & alf & $7-8$ & $\mathrm{mh}$ & es & $\mathrm{a}$ & 3.00 & e & ate \\
\hline $\begin{array}{c}\text { Nitzschia tryblionella var. } \\
\text { ambigua Grunow }\end{array}$ & B & - & - & - & - & hl & - & $\mathrm{b}$ & 2.00 & - & - \\
\hline $\begin{array}{c}\text { Nitzschia vermicularis } \\
\text { (Kützing) Hantzsch in } \\
\text { Rabenhorst }\end{array}$ & P-B & - & str & alf & - & $\mathrm{i}$ & - & b & 2.20 & $\mathrm{~m}$ & - \\
\hline
\end{tabular}




\begin{tabular}{|c|c|c|c|c|c|c|c|c|c|c|c|}
\hline Taxa & Hab & $\mathbf{T}$ & $\mathbf{O x y}$ & pH & pH-range & Sal & $\mathbf{D}$ & Sap & $\mathbf{S}$ & Tro & $\begin{array}{l}\text { Aut- } \\
\text { Het }\end{array}$ \\
\hline $\begin{array}{l}\text { Odontidium mesodon } \\
\text { (Kützing) Kützing }\end{array}$ & B & cool & st-str & neu & 6.9 & $\mathrm{hb}$ & SX & $\mathrm{X}-\mathrm{O}$ & 0.40 & ot & ats \\
\hline $\begin{array}{l}\text { Pinnularia brauniana } \\
\text { (Grunow) Studnicka }\end{array}$ & P-B & - & - & acf & - & $\mathrm{i}$ & - & $\mathrm{x}$ & 0.20 & ot & - \\
\hline $\begin{array}{l}\text { Pinnularia hemiptera } \\
\text { Brébisson ex Greville }\end{array}$ & B & - & str & ind & - & $\mathrm{i}$ & - & $\mathrm{o}$ & 1.00 & ot & - \\
\hline $\begin{array}{c}\text { Pinnularia rhombarea var. } \\
\text { halophila Krammer }\end{array}$ & B & - & - & - & - & - & - & - & - & - & - \\
\hline $\begin{array}{l}\text { Pinnularia viridis } \\
\text { (Nitzsch) Ehrenberg }\end{array}$ & P-B & temp & st-str & ind & 7.1 & $\mathrm{i}$ & es & $\mathrm{x}$ & 0.30 & o-e & ate \\
\hline $\begin{array}{l}\text { Placoneis elginensis } \\
\text { (W.Gregory) E.J.Cox }\end{array}$ & B & - & st-str & ind & $7-9$ & $\mathrm{i}$ & SX & o-b & 1.40 & me & ate \\
\hline $\begin{array}{c}\text { Planothidium lanceolatum } \\
\text { (Brébisson ex Kützing) } \\
\text { Lange-Bertalot }\end{array}$ & P-B & warm & st-str & alf & $7.5-8.1$ & $\mathrm{i}$ & SX & $\mathrm{b}-\mathrm{o}$ & 1.60 & $\mathrm{e}$ & ate \\
\hline $\begin{array}{c}\text { Platessa salinarum } \\
\text { (Grunow) Lange-Bertalot }\end{array}$ & P-B & - & st-str & ind & - & $\mathrm{mh}$ & - & $\mathrm{b}$ & 2.10 & me & ate \\
\hline $\begin{array}{c}\text { Pleurosigma elongatum } \\
\text { W.Smith }\end{array}$ & $\mathrm{B}$ & - & - & alf & - & $\mathrm{mh}$ & - & $\mathrm{b}$ & 2.00 & - & - \\
\hline $\begin{array}{c}\text { Psammothidium } \\
\text { semiapertum (Hustedt) } \\
\text { Aboal } \\
\end{array}$ & B & - & - & - & - & - & - & - & - & - & - \\
\hline $\begin{array}{l}\text { Rhoicosphenia abbreviata } \\
\text { (C.Agardh) Lange-Bertalot }\end{array}$ & B & - & st-str & alf & 6.7 & $\mathrm{i}$ & es & $\mathrm{o}-\mathrm{a}$ & 1.90 & me & ate \\
\hline $\begin{array}{c}\text { Rhopalodia gibba } \\
\text { (Ehrenberg) Otto Müller }\end{array}$ & B & temp & - & alf & $6.2-9.0$ & $\mathrm{i}$ & es & o-b & 1.40 & $\mathrm{o}-\mathrm{m}$ & - \\
\hline $\begin{array}{c}\text { Rhopalodia gibba var. } \\
\text { ventricosa (Kützing) }\end{array}$ & B & temp & - & alf & $6.2-9.0$ & $\mathrm{i}$ & es & o-b & 1.40 & - & - \\
\hline
\end{tabular}

APPLIED ECOLOGY AND ENVIRONMENTAL RESEARCH 16(6):7799-7831.

http://www.aloki.hu • ISSN 15891623 (Print) • ISSN 17850037 (Online)

DOI: http://dx.doi.org/10.15666/aeer/1606_77997831

( 2018 , ALÖKI Kft., Budapest, Hungary 


\begin{tabular}{|c|c|c|c|c|c|c|c|c|c|c|c|}
\hline Taxa & Hab & $\mathbf{T}$ & Oxy & pH & pH-range & Sal & $\mathbf{D}$ & Sap & $\mathbf{S}$ & Tro & $\begin{array}{l}\text { Aut- } \\
\text { Het }\end{array}$ \\
\hline \multicolumn{12}{|l|}{ H.Peragallo \& M.Peragallo } \\
\hline $\begin{array}{l}\text { Sellaphora submuralis } \\
\text { (Hustedt) C.E.Wetzel, } \\
\text { L.Ector, B.Van de Vijver, } \\
\text { Compère \& D.G.Mann }\end{array}$ & B & - & - & - & - & - & es & $\mathrm{x}$ & 0.10 & - & - \\
\hline $\begin{array}{l}\text { Stauroneis phoenicenteron } \\
\text { (Nitzsch) Ehrenberg }\end{array}$ & P-B & temp & st-str & ind & 7.3 & $\mathrm{i}$ & es & o & 1.30 & me & ate \\
\hline $\begin{array}{c}\text { Staurosira venter } \\
\text { (Ehrenberg) Cleve \& } \\
\text { J.D.Möller }\end{array}$ & P-B & warm & st-str & alf & $5.5-9.0$ & $\mathrm{i}$ & sx & o & 1.30 & me & ate \\
\hline $\begin{array}{c}\text { Surirella brebissonii } \\
\text { Krammer \& Lange- } \\
\text { Bertalot }\end{array}$ & B & - & st-str & alf & - & $\mathrm{i}$ & - & b-o & 1.70 & - & - \\
\hline $\begin{array}{c}\text { Surirella elegans } \\
\text { Ehrenberg }\end{array}$ & P-B & - & str & alf & - & $\mathrm{i}$ & - & $\mathrm{o}$ & 1.00 & me & - \\
\hline $\begin{array}{c}\text { Surirella librile } \\
\text { (Ehrenberg) Ehrenberg }\end{array}$ & B & - & st-str & alf & 8.0 & $\mathrm{i}$ & - & $\mathrm{b}$ & 2.10 & $\mathrm{e}$ & ate \\
\hline Surirella patella Kützing & $\mathrm{B}$ & - & - & - & - & $\mathrm{mh}$ & - & - & - & - & - \\
\hline Synedra familiaris Kützing & $\mathrm{B}$ & - & - & ind & 7.7 & $\mathrm{i}$ & $\mathrm{sx}$ & $\mathrm{o}-\mathrm{b}$ & 1.50 & - & - \\
\hline $\begin{array}{l}\text { Synedra rumpens var. } \\
\text { scotica Grunow in Van } \\
\text { Heurck }\end{array}$ & B & - & - & - & - & $\mathrm{i}$ & es & b-o & 1.60 & - & - \\
\hline $\begin{array}{c}\text { Tabularia fasciculata } \\
\text { (C.Agardh) D.M.Williams } \\
\text { \& Round }\end{array}$ & P-B & - & st & ind & - & $\mathrm{mh}$ & es & $\mathrm{b}-\mathrm{a}$ & 2.50 & $\mathrm{e}$ & ate \\
\hline $\begin{array}{l}\text { Tryblionella hungarica } \\
\text { (Grunow) Frenguelli }\end{array}$ & P-B & - & - & alf & - & $\mathrm{mh}$ & $\mathrm{sp}$ & a-o & 2.90 & $\mathrm{e}$ & ate \\
\hline Tryblionella levidensis & P-B & - & st-str & ind & - & $\mathrm{mh}$ & $\mathrm{sp}$ & a-o & 2.60 & $\mathrm{e}$ & ate \\
\hline
\end{tabular}

APPLIED ECOLOGY AND ENVIRONMENTAL RESEARCH 16(6):7799-7831.

http://www.aloki.hu • ISSN 15891623 (Print) • ISSN 17850037 (Online)

DOI: http://dx.doi.org/10.15666/aeer/1606_77997831

○ 2018, ALÖKI Kft., Budapest, Hungary 


\begin{tabular}{c|c|c|c|c|c|c|c|c|c|c|c}
\hline Taxa & Hab & T & Oxy & pH & pH-range & Sal & D & Sap & S & Tro & $\begin{array}{c}\text { Aut- } \\
\text { Het }\end{array}$ \\
\hline W.Smith & & & & & & & & & & \\
\hline $\begin{array}{c}\text { Tryblionella navicularis } \\
\text { (Brébisson) Ralfs in } \\
\text { Pritchard }\end{array}$ & B & - & - & - & - & mh & - & - & - & - \\
\hline $\begin{array}{c}\text { Ulnaria amphirhynchus } \\
\text { (Ehrenberg) Compère \& } \\
\begin{array}{c}\text { Bukhtiyarova in } \\
\text { Bukhtiyarova \& Compère }\end{array}\end{array}$ & P-B & - & - & alf & - & i & es & b & 2.00 & o-m \\
\hline $\begin{array}{c}\text { Ulnaria ulna } \text { (Nitzsch) } \\
\text { Compère }\end{array}$ & P-B & temp & st-str & ind & $5.0-9.2$ & i & es & b & 2.25 & o-e \\
\hline
\end{tabular}

\begin{tabular}{|c|c|c|c|c|c|c|c|c|c|c|c|}
\hline \multicolumn{12}{|l|}{ Euglenozoa } \\
\hline Euglena deses Ehrenberg & P-B, S & warm & st-str & ind & $6.0-8.3$ & $\mathrm{mh}$ & - & $\mathrm{b}$ & 2.20 & - & - \\
\hline $\begin{array}{c}\text { Euglena sanguinea } \\
\text { Ehrenberg }\end{array}$ & P-B & - & st-str & $\operatorname{acf}$ & $5.5-6.7$ & $\mathrm{i}$ & - & o & 1.25 & - & - \\
\hline $\begin{array}{c}\text { Lepocinclis acus } \\
\text { (O.F.Müller) B.Marin \& } \\
\text { Melkonian in Marin et al. }\end{array}$ & $\mathrm{P}$ & eterm & st & ind & $7.2-8.2$ & $\mathrm{i}$ & - & $\mathrm{b}$ & 2.20 & - & - \\
\hline Phacus acutus Pochmann & P-B & - & st & ind & $7.0-7.2$ & $\mathrm{i}$ & - & a-o & 2.80 & - & - \\
\hline $\begin{array}{c}\text { Phacus orbicularis } \\
\text { K.Hübner }\end{array}$ & P-B & - & st-str & ind & 7.0 & $\mathrm{i}$ & - & $\mathrm{b}-\mathrm{a}$ & 2.45 & - & - \\
\hline $\begin{array}{l}\text { Phacus triqueter } \\
\text { (Ehrenberg) Perty }\end{array}$ & P-B & - & st-str & - & - & $\mathrm{i}$ & - & $\mathrm{b}-\mathrm{a}$ & 2.50 & - & - \\
\hline \multicolumn{12}{|l|}{ Chlorophyta } \\
\hline $\begin{array}{c}\text { Ankistrodesmus spiralis } \\
\text { (W.B.Turner) } \\
\text { Lemmermann }\end{array}$ & $\mathrm{P}$ & - & - & - & - & - & - & $\mathrm{b}$ & 2.10 & - & - \\
\hline
\end{tabular}




\begin{tabular}{|c|c|c|c|c|c|c|c|c|c|c|c|}
\hline Taxa & Hab & $\mathbf{T}$ & Oxy & pH & pH-range & Sal & D & Sap & $\mathbf{S}$ & Tro & $\begin{array}{l}\text { Aut- } \\
\text { Het }\end{array}$ \\
\hline $\begin{array}{c}\text { Botryococcus braunii } \\
\text { Kützing }\end{array}$ & P-B & - & st & ind & - & $\mathrm{i}$ & - & o-b & 1.50 & - & - \\
\hline $\begin{array}{c}\text { Bulbochaete intermedia De } \\
\text { Bary ex Hirn }\end{array}$ & B & - & - & - & - & - & - & b-o & 1.70 & - & - \\
\hline $\begin{array}{c}\text { Bulbochaete nana Wittrock } \\
\text { ex Hirn }\end{array}$ & B & - & - & - & - & - & - & o & 1.30 & - & - \\
\hline $\begin{array}{l}\text { Chlorococcum infusionum } \\
\text { (Schrank) Meneghini }\end{array}$ & $\mathrm{P}, \mathrm{S}$ & - & st & - & - & - & - & $\mathrm{a}-\mathrm{o}$ & 2.70 & - & - \\
\hline $\begin{array}{l}\text { Cladophora glomerata } \\
\text { (Linnaeus) Kützing }\end{array}$ & P-B & - & st-str & alf & $7.5-8.5$ & $\mathrm{i}$ & - & o-a & 1.90 & - & - \\
\hline $\begin{array}{l}\text { Coelastrum microporum } \\
\text { Nägeli in A.Braun }\end{array}$ & P-B & - & st-str & ind & - & $\mathrm{i}$ & - & $\mathrm{b}$ & 2.30 & - & - \\
\hline $\begin{array}{l}\text { Desmodesmus tropicus } \\
\text { (W.B.Crow) E.Hegewald }\end{array}$ & $\mathrm{P}$ & - & st & - & - & - & - & - & - & - & - \\
\hline $\begin{array}{l}\text { Geminella ellipsoidea } \\
\text { (Prescott) G.M.Smith }\end{array}$ & $\mathrm{P}$ & - & - & - & - & - & - & - & - & - & - \\
\hline $\begin{array}{c}\text { Messastrum gracile } \\
\text { (Reinsch) T.S.Garcia in } \\
\text { T.S.Garcia et al. }\end{array}$ & $\mathrm{P}$ & - & - & - & - & - & - & o-a & 1.90 & - & - \\
\hline $\begin{array}{l}\text { Oedogonium obtruncatum } \\
\text { Wittrock ex Hirn }\end{array}$ & - & - & - & - & - & - & - & - & - & - & - \\
\hline $\begin{array}{c}\text { Raphidocelis subcapitata } \\
\text { (Korshikov) Nygaard, } \\
\text { Komárek, J.Kristiansen \& } \\
\text { O.M.Skulberg }\end{array}$ & P-B & - & st-str & - & - & $\mathrm{i}$ & - & $o-b$ & 1.50 & - & - \\
\hline $\begin{array}{l}\text { Scenedesmus armatus } \\
\text { (Chodat) Chodat }\end{array}$ & P-B & - & st-str & - & - & - & - & $\mathrm{b}$ & 2.20 & - & - \\
\hline $\begin{array}{c}\text { Scenedesmus quadrispina } \\
\text { Chodat }\end{array}$ & P-B & - & st-str & - & - & - & - & o-a & 1.90 & - & - \\
\hline Volvox aureus Ehrenberg & $\mathrm{P}$ & - & st & - & - & $\mathrm{i}$ & - & $\mathrm{b}$ & 2.20 & - & - \\
\hline
\end{tabular}




\begin{tabular}{|c|c|c|c|c|c|c|c|c|c|c|c|}
\hline Taxa & Hab & $\mathbf{T}$ & Oxy & pH & pH-range & Sal & $\mathbf{D}$ & Sap & $\mathbf{S}$ & Tro & $\begin{array}{l}\text { Aut- } \\
\text { Het }\end{array}$ \\
\hline \multicolumn{12}{|l|}{ Charophyta } \\
\hline $\begin{array}{c}\text { Chara aspera } \\
\text { C.L.Willdenow }\end{array}$ & B & - & - & - & - & - & - & o & 1.20 & - & - \\
\hline Chara vulgaris L. & $\mathrm{B}$ & - & st-str & - & - & - & - & o & 1.10 & - & - \\
\hline $\begin{array}{c}\text { Cosmarium bioculatum } \\
\text { var. excavatum Gutwinski }\end{array}$ & P-B & - & st-str & ind & - & $\mathrm{hb}$ & - & $\mathrm{X}-\mathrm{O}$ & 0.50 & $\mathrm{~m}$ & - \\
\hline $\begin{array}{l}\text { Cosmarium botrytis } \\
\text { Meneghini ex Ralfs }\end{array}$ & P-B & - & st-str & ind & - & $\mathrm{i}$ & - & $\mathrm{o}-\mathrm{a}$ & 1.90 & $\mathrm{~m}$ & - \\
\hline $\begin{array}{c}\text { Cosmarium clepsydra var. } \\
\text { dissimile (Raciborski) } \\
\text { Krieger \& Gerloff }\end{array}$ & - & - & - & - & - & - & - & - & - & $\mathrm{m}$ & - \\
\hline $\begin{array}{l}\text { Cosmarium obtusatum } \\
\text { (Schmidle) Schmidle }\end{array}$ & B & - & - & ind & - & $\mathrm{i}$ & - & o & 1.30 & me & - \\
\hline $\begin{array}{c}\text { Cosmarium phaseolus } \\
\text { Brébisson ex Ralfs } \\
\end{array}$ & $\mathrm{B}$, aer & - & - & acf & - & $\mathrm{i}$ & - & - & - & $\mathrm{m}$ & - \\
\hline $\begin{array}{c}\text { Cosmarium subcrenatum } \\
\text { var. subdivaricatum } \\
\text { Gutwinski }\end{array}$ & $\mathrm{B}$, aer & - & aer & acf & - & - & - & o & 1.10 & $\mathrm{~m}$ & - \\
\hline $\begin{array}{c}\text { Cosmarium } \\
\text { tetraophthalmum } \\
\text { Brébisson ex Ralfs }\end{array}$ & P-B & - & st-str & ind & - & - & - & - & - & $\mathrm{m}$ & - \\
\hline $\begin{array}{c}\text { Cosmarium wittrockii } \\
\text { P.Lundell }\end{array}$ & B & - & - & acf & - & - & - & - & - & $\mathrm{m}$ & - \\
\hline $\begin{array}{c}\text { Mougeotia genuflexa } \\
\text { (Roth) C.Agardh }\end{array}$ & B & - & - & - & - & - & - & $\mathrm{O}-\mathrm{X}$ & 1.00 & - & - \\
\hline $\begin{array}{l}\text { Nitella hyalina (De } \\
\text { Candolle) C.Agardh }\end{array}$ & B & - & - & - & - & - & - & - & - & - & - \\
\hline $\begin{array}{l}\text { Nitella tenuissima } \\
\text { (Desvaux) Kützing }\end{array}$ & $\mathrm{B}$ & - & st & - & - & - & - & $\mathrm{x}-\mathrm{b}$ & 0.80 & - & - \\
\hline Spirogyra longata & $\mathrm{B}$ & - & st & - & - & - & - & $\mathrm{o}$ & 1.00 & - & - \\
\hline
\end{tabular}




\begin{tabular}{|c|c|c|c|c|c|c|c|c|c|c|c|}
\hline Taxa & Hab & $\mathbf{T}$ & Oxy & pH & pH-range & Sal & D & Sap & $\mathbf{S}$ & Tro & $\begin{array}{l}\text { Aut- } \\
\text { Het }\end{array}$ \\
\hline \multicolumn{12}{|l|}{ (Vaucher) Kuetzing } \\
\hline $\begin{array}{c}\text { Staurastrum boreale West } \\
\text { \& G.S.West }\end{array}$ & B & - & - & ind & - & - & - & - & - & $\mathrm{m}$ & - \\
\hline $\begin{array}{l}\text { Staurastrum hexacerum } \\
\text { var. hexacerum Wittrock }\end{array}$ & $\mathrm{P}$ & - & - & acf & - & - & - & o-b & 1.50 & $\mathrm{~m}$ & - \\
\hline $\begin{array}{c}\text { Staurastrum manfeldtii var. } \\
\text { ucrainicum (Palamar- } \\
\text { Mordvintseva) Petlovany }\end{array}$ & B & - & - & ind & - & - & - & o-a & 1.80 & $\mathrm{~m}$ & - \\
\hline $\begin{array}{l}\text { Staurastrum retusum var. } \\
\text { boreale West \& G.S.West }\end{array}$ & B & - & - & ind & - & - & - & - & - & me & - \\
\hline $\begin{array}{c}\text { Zygnema pectinatum } \\
\text { (Vaucher) C.Agardh in } \\
\text { Liljeblad }\end{array}$ & B & - & st-str & - & - & oh & - & o & 1.00 & - & - \\
\hline $\begin{array}{c}\text { Zygnema ralfsii (Hassall) } \\
\text { De Bary }\end{array}$ & B & - & - & - & - & - & - & o & 1.00 & - & - \\
\hline
\end{tabular}

Abbreviation of the ecological groups of indicators:

Habitat preferences: $\mathrm{P}$ - planktonic, $\mathrm{P}-\mathrm{B}$ - planktonic-benthic, B - benthic, wide range, need some substrate; $\mathrm{S}$ - soil.

Water Temperature preferences: warm - warm-water inhabitants; cool - cool-water inhabitants; temp - temperate water temperature inhabitants or indifferent; eterm - eurythermic.

Oxygenation indicators: st - standing water, str - streaming water, st-str - low streaming water.

Salinity: hb - oligohalobes-halophobes, i - oligohalobes-indifferents, $\mathrm{mh}$ - mesohalobes, hl - halophiles.

Acidity ( $\mathrm{pH})$ degree indicators: alb - alkalibiontes; alf - alkaliphiles, ind - indifferents; acf - acidophiles.

Watanabe indicators of organic pollution (Watanabe et al., 1986): sx - saproxen; es - eurysaprob; sp - saprophil.

Nitrogen uptake metabolism indicators: ats - nitrogen-autotrophic taxa, tolerating very small concentrations of organically bound nitrogen; ate - nitrogen-autotrophic taxa, tolerating elevated concentrations of organically bound nitrogen; hne - facultatively nitrogen-heterotrophic taxa, needing periodically elevated concentrations of organically bound nitrogen; hce - obligately nitrogen-heterotrophic taxa, needing continuously elevated concentrations of organically bound nitrogen.

Trophic state indicators: ot - oligotraphentic; o-m - oligo-mesotraphentic; m - mesotraphentic; me - meso-eutraphentic; e - eutraphentic; o-e - oligo- to eutraphentic (hypereutraphentic) 
Fig. $2 a$ shows distribution of algal species richness over taxonomic Divisions. Can be seen that only Bacillariophyta species that cut off by standard deviation line were strongly prevail in the communities of the lake. Algae from the indicators list are prefer benthic and bentho-planktonic life still (that cut off by Standard Deviation (stdev) line) but few planktonic inhabitants were also revealed (Fig. 2b). In the water temperature indicators temperate temperature species were strongly prevail and were cut off by stdev line but significant number of indicators of warm water were recognized also (Fig. 2c). Indicators of saturation of water by oxygen show middle oxygenation with indicators of low-streaming waters that cut off by stdev line (Fig. 2d).
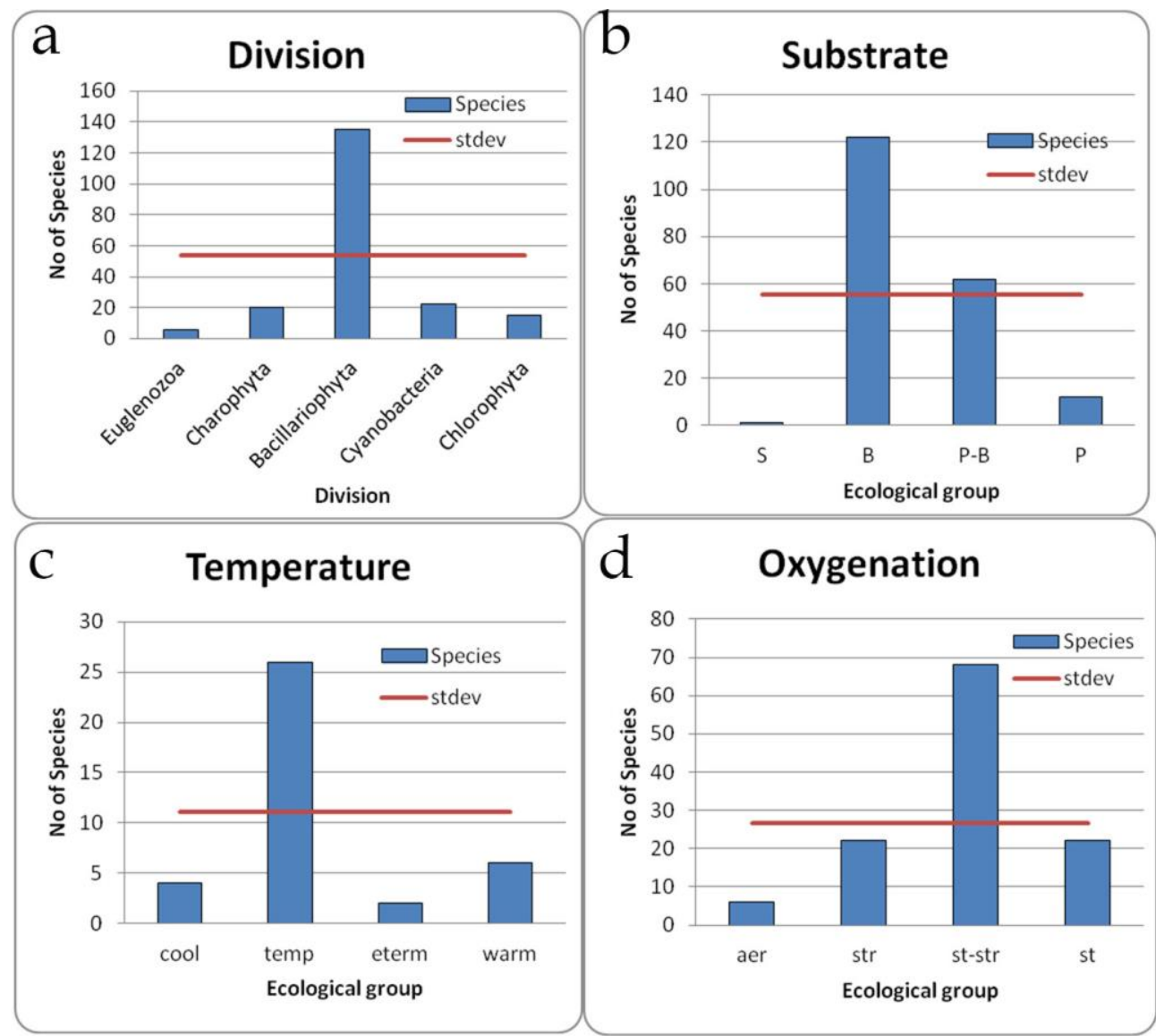

Figure 2. Distribution of taxonomic and algal bioindication groups of substrate preferences, water temperature and oxygenation in the Alakol Lake in 2015-2017. Abbreviation: a, Distribution of species over taxonomic Divisions. $b$, Habitat preferences: $P$ - planktonic, $P-B-$ planktonic-benthic, $B$ - benthic, wide range, need some substrate; $S$ - soil. $c$, Water Temperature preferences: warm - warm-water inhabitants; cool-cool-water inhabitants; temp - temperate water temperature inhabitants or indifferent; eterm - eurythermic. d, Oxygenation indicators: st-standing water, str-streaming water, st-str-low streaming water

Indicators of water $\mathrm{pH}$ reflect low alkaline water in the Alakol Lake with prevailing of alkaliphilic and indifferents ecological groups (Fig. 3a) that cut off by stdev line. In the salinity indicators the indifferents group, which cut off by stdev line, was strongly prevail and reflect low saline waters in the studied lake (Fig. 3b). It is very important to assess the organic pollution load in the Alakol Natural Reserve. So, we implemented 
two indicator systems for this analysis. Indicators of Watanabe are all diatom algae only and in studied lake community they are strongly prevail. Fig. $3 c$ shows distribution of Watanabe indicator species that help us to assess the lake waters as low organically polluted with prevailing of eurysaprobes, indicators of middle pollution of water, and saproxenes that are indicates of clear unpolluted water. Both these group are cut off by stdev line. Indicators of organic pollution according species-specific Saprobity index $S$ shows distribution over all five classes of water quality (Barinova, 2017b) with prevailing of Class 2 and 3 which are cut off by stdev line (Fig. 3d). So, analysis of organic pollution demonstrated the coincidence of estimates in both systems.

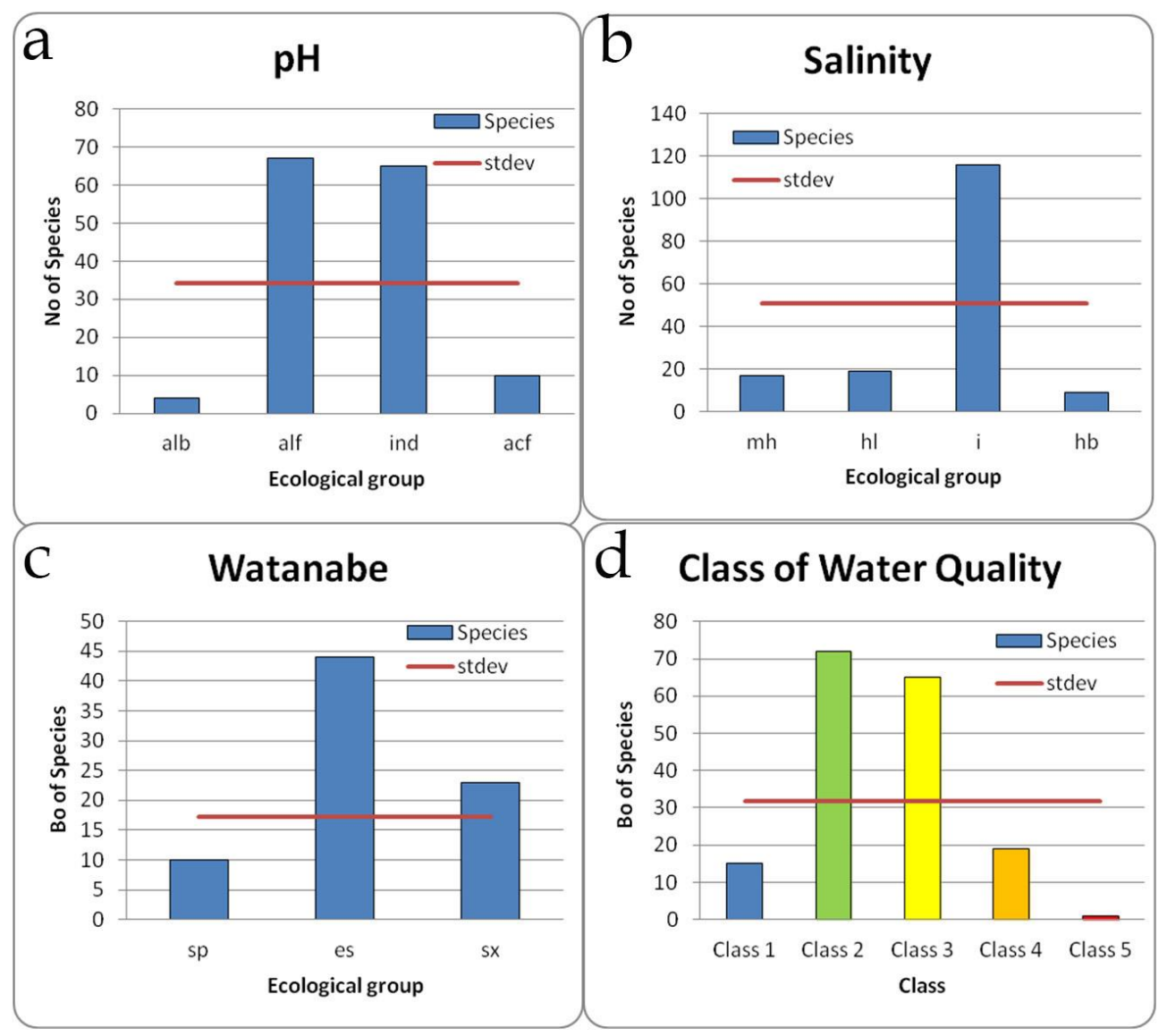

Figure 3. Distribution of algal bioindication groups of water $\mathrm{pH}$, salinity, organic pollution according Watanabe, and Water Quality Class according Saprobity index $S$ in the Alakol Lake in 2015-2017. Abbreviation: a, Acidity $(\mathrm{pH})$ degree indicators: alb - alkalibiontes; alf alkaliphiles, ind-indifferents; acf-acidophiles. $b$, Salinity: $h b$-oligohalobes-halophobes, $i-$ oligohalobes-indifferents, $m h$-mesohalobes, $h l$-halophiles. $c$, Watanabe indicators of organic pollution (Watanabe et al., 1986): sx-saproxen; es - eurysaprob; sp - saprophil. d, Class of Water Quality according EU color codes

Trophic state of the Alakol Lake was assessed with species indicators as oligo-to mesotrophic (Fig. 4a). Remarkable that stdev line cut off five indicator groups from oligotrophic to eutrophic species that represents wide range of tropic state of the lake. In this case, we implemented polynomial trend line construction and it revealed two major trophic state of the lake water - oligotrophic and meso-eutrophic with two peaks. It can be results of some pollution input from point sources which were not dispersed over the 
whole lake. Nutrition type of algal species in the Alakol Lake (Fig. 4b) is strongly autotrophic. It is good conclusion for the algal communities in the Natural Reserve lake.
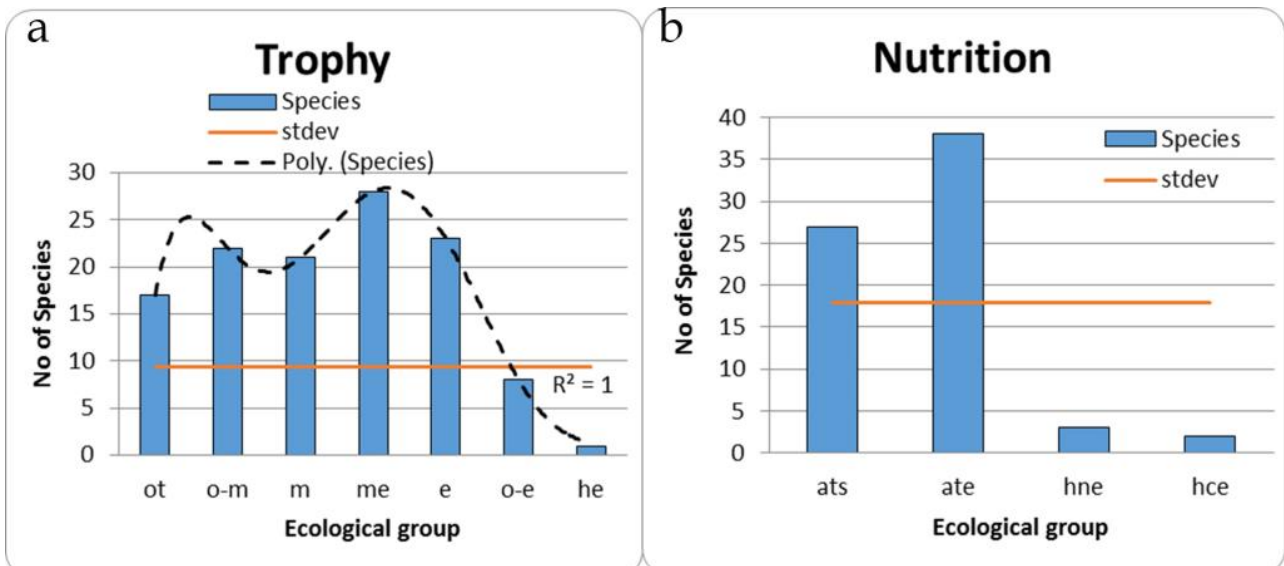

Figure 4. Distribution of algal bioindication groups of trophic state and algal nutrition type in the Alakol Lake in 2015-2017. Abbreviation: a, Trophic state indicators: ot - oligotraphentic; $o$-m-oligo-mesotraphentic; $m$-mesotraphentic; me - meso-eutraphentic; $e$-eutraphentic; $o$ $e$-oligo- to eutraphentic (hypereutraphentic). $b$, Nitrogen uptake metabolism indicators: ats nitrogen-autotrophic taxa, tolerating very small concentrations of organically bound nitrogen; ate - nitrogen-autotrophic taxa, tolerating elevated concentrations of organically bound nitrogen; hne - facultatively nitrogen-heterotrophic taxa, needing periodically elevated concentrations of organically bound nitrogen; hce - obligately nitrogen-heterotrophic taxa, needing continuously elevated concentrations of organically bound nitrogen

This conclusion led us to conduct a spatial bioindication analysis in order to determine the sources of organic pollution. In this purpose, we divided all indicator species that were found in the sampling points to three sampling areas from north to south along the lake coast: Kamyskala, Akshi, and Koktuma. Each area have its species diversity, in Kamyskala 79 species, in Akshi 19 species, and in Koktuma 118 algal species. Fig. $5 a$ shows distribution of species richness in taxonomic Divisions over three lake areas. Diatoms prevail in all communities but Kamyskala looks like more diverse because include species from all five revealed Divisions. Benthic inhabitants are prevail in Akshi area (Fig. 5b). Temperature indicators are more complicated in the southern area Koktuma, but warm water indicators were found in northern area Kamyskala together with cool water indicators (Fig. 5c). It can be if the environment of this area was influenced by two different factors such as warming of water in the shallow bays and the influence of underground river flow. Waters of the lake were better oxygen saturated in the Akshi area with prevailing of streaming ecological group, but aerophiles were revealed in both northern and southern areas also (Fig. 5d).

Distribution of species-indicators of water $\mathrm{pH}$ (Fig. 6a) revealed not only more alkaline waters in the Akshi area, but also influence of the Zhamanty permanent river inflow with alkalibionts indicators community in the area of Koktuma. In the Akshi area algal communities, we revealed also few indicators of water acidification. Water salinity indicators distribution (Fig. 6b) show similarity of Akshi and Kamyskala areas waters where indicators of indifferents group are prevail. Kamyskala area waters were more complicated in salinity indicators content. Here we can see not only halophobe species, 
which prefer fresh water, but also oligohalobes-halophiles and even mesohalobes that are indicators of low saline to mineralized waters.
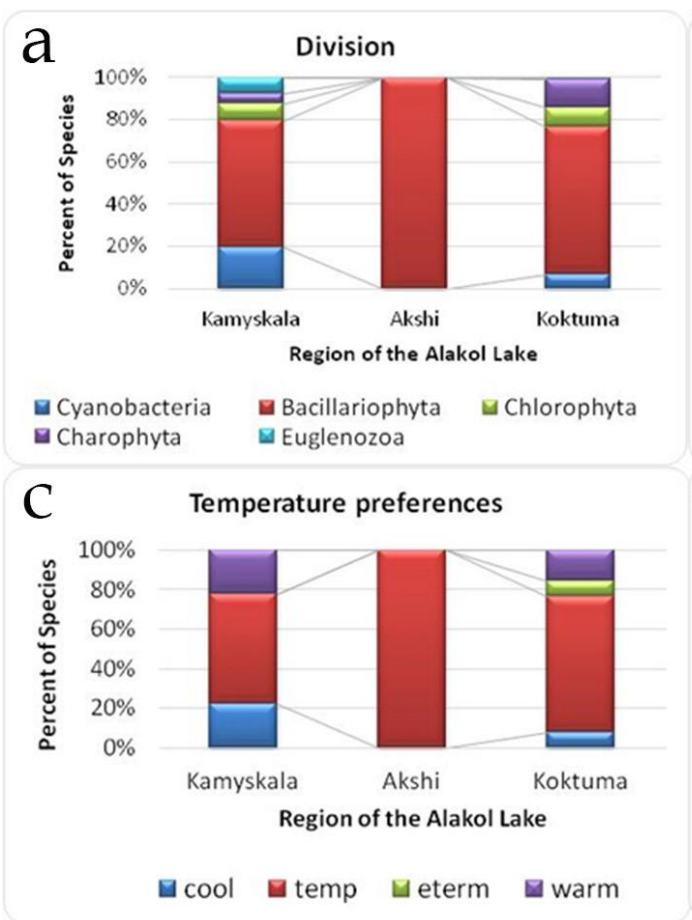
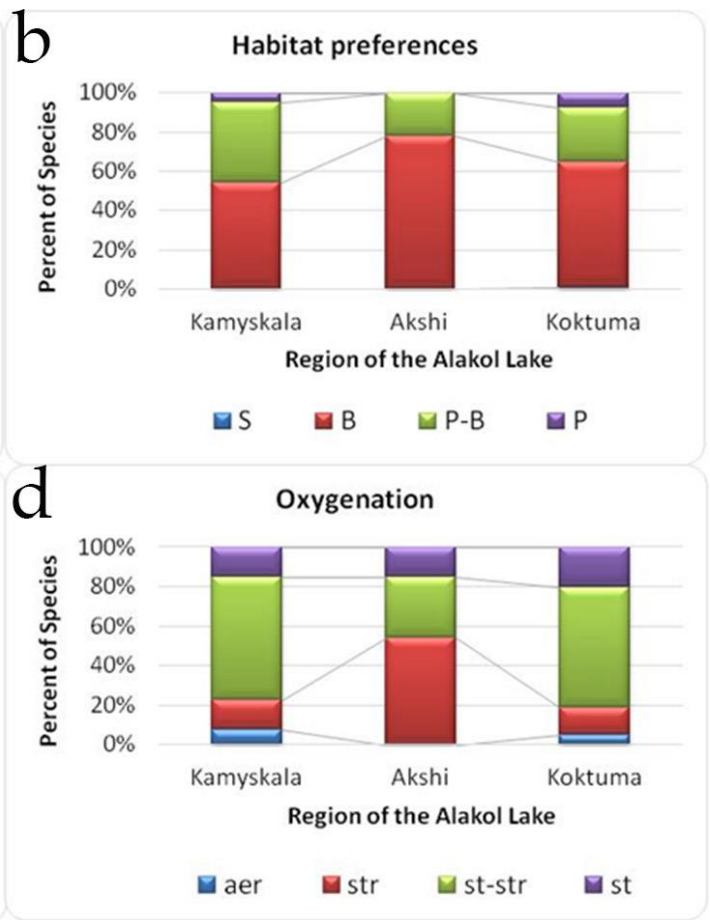

Figure 5. Distribution of algal species in taxonomic Divisions and ecological indicators of substrate, water temperature and oxygenation preferences for the Alakol Lake sampling areas Kamyskala, Akshi, and Koktuma in 2015-2017. Abbreviation: a, Distribution of species over taxonomic Divisions. $b$, Habitat preferences: $P$ - planktonic, $P-B$ - planktonic-benthic, $B-$ benthic, wide range, need some substrate; $S$ - soil. c, Water Temperature preferences: warmwarm-water inhabitants; cool - cool-water inhabitants; temp - temperate water temperature inhabitants or indifferent; eterm - eurythermic. d, Oxygenation indicators: st - standing water, str-streaming water, st-str-low streaming water
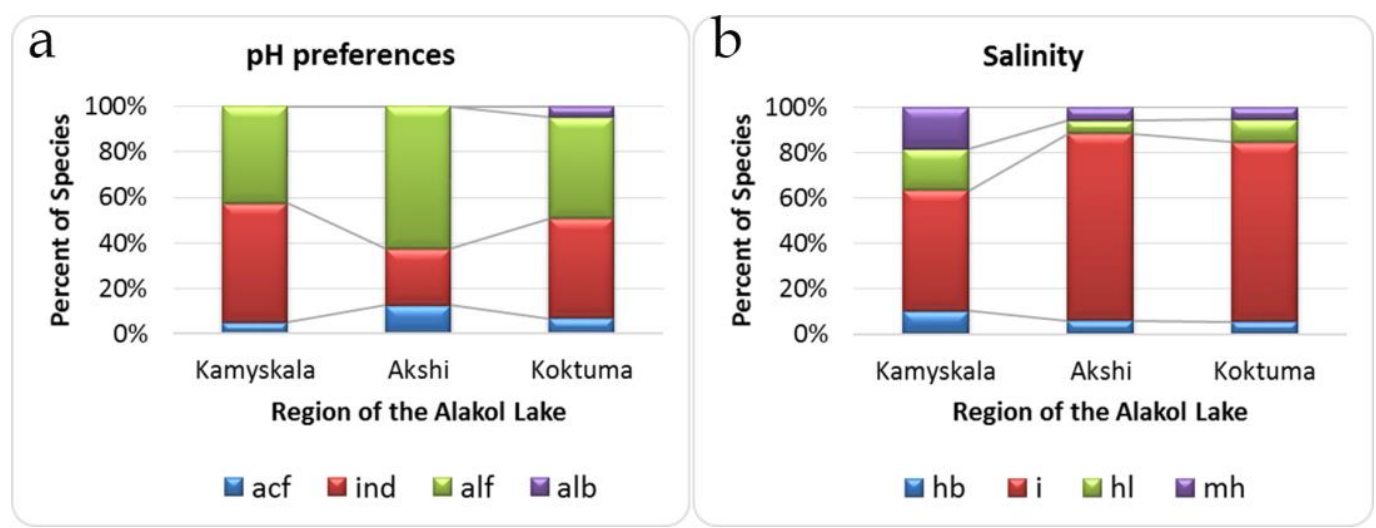

Figure 6. Distribution of algal indicators of water $p H$, oxygenation, temperature, and salinity in the Alakol Lake sampling areas Kamyskala, Akshi, and Koktuma in 2015-2017. Abbreviation: a, Acidity $(\mathrm{pH})$ degree indicators: alb - alkalibiontes; alf - alkaliphiles, ind-indifferents; acf acidophiles. b, Salinity: $h b$ - oligohalobes-halophobes, $i$ - oligohalobes-indifferents, $m h-$ mesohalobes, hl-halophiles 
It was very important to reveal organic pollution indicators distribution along the lake surface. Fig. $7 a$ shows increasing of clear water indicators saproxenes and decreasing of polluted water indicators saprophiles from north to south. This distribution is demonstrated self-purification tendency increasing from Kamyskala area to Koktuma in accordance prevailing diatom group of algae in the Alakol Lake communities. Similar trend of self-purification processes are demonstrated by five classes of saprobily indicators distribution. Can be seen that percent of clear water indicators of Class 1 and 2 is increased from Kamyskala to Koktuma area when indicators of Class 4 and 5 are decreased significantly (Fig. 7b).
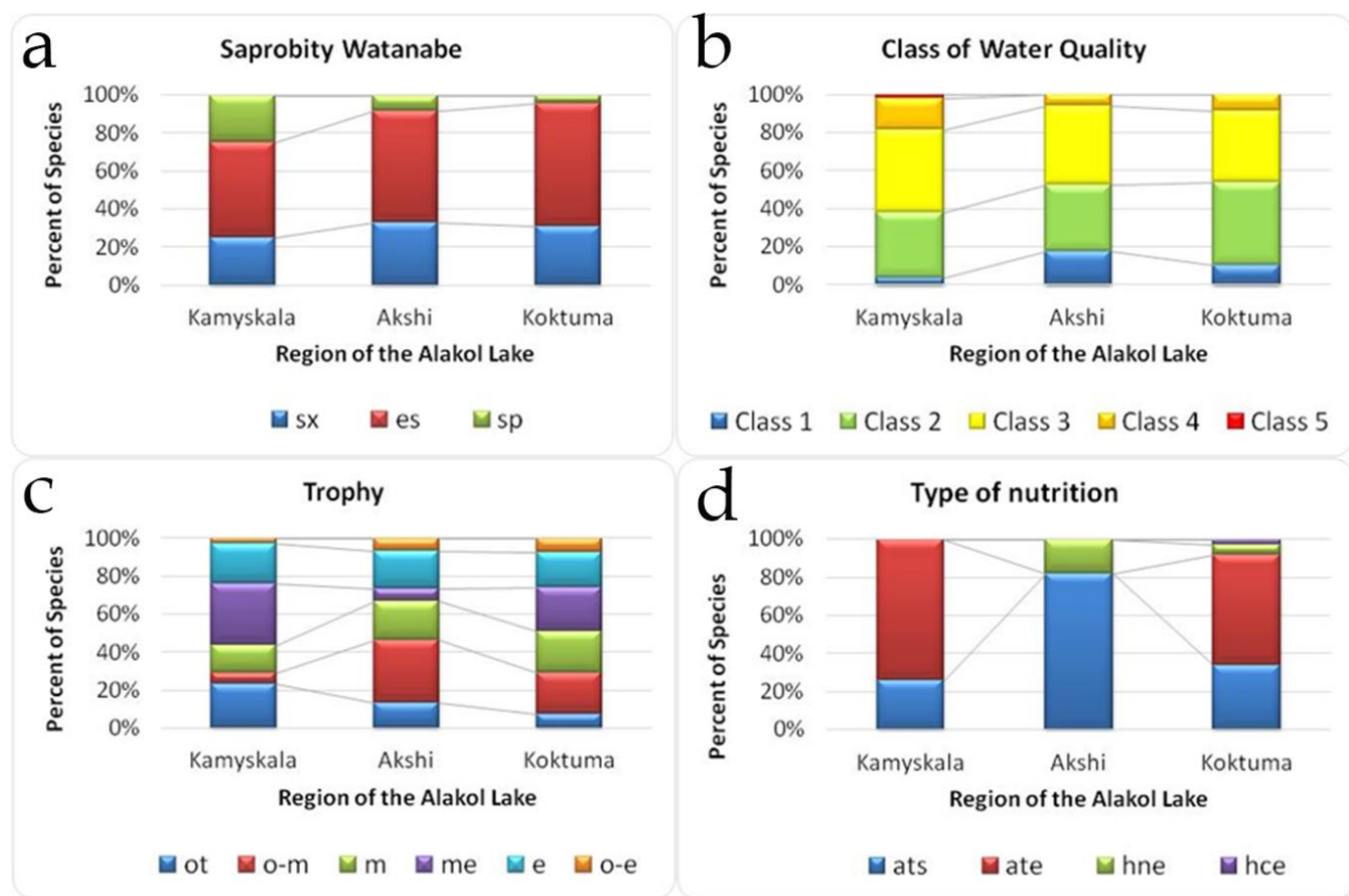

Figure 7. Distribution of algal indicators of organic pollution according Watanabe, Class of Water Quality according Saprobity index S, trophic state, and algal nutrition type in the Alakol Lake sampling areas Kamyskala, Akshi, and Koktuma in 2015-2017. Abbreviation: a, Watanabe indicators of organic pollution (Watanabe et al., 1986): sx - saproxen; es - eurysaprob; sp saprophil. b, Class of Water Quality according EU color codes. c, Trophic state indicators: ot oligotraphentic; $o$-m - oligo-mesotraphentic; $m$-mesotraphentic; me - meso-eutraphentic; $e-$ eutraphentic; o-e - oligo- to eutraphentic (hypereutraphentic). $d$, Nitrogen uptake metabolism indicators: ats - nitrogen-autotrophic taxa, tolerating very small concentrations of organically bound nitrogen; ate - nitrogen-autotrophic taxa, tolerating elevated concentrations of organically bound nitrogen; hne - facultatively nitrogen-heterotrophic taxa, needing periodically elevated concentrations of organically bound nitrogen; hce - obligately nitrogenheterotrophic taxa, needing continuously elevated concentrations of organically bound nitrogen

It is very interesting to reveal distribution of trophic indicators along the studied lake (Fig. 7c). It turned out that indicators of meso- and eutrophic waters are increased from north to south whereas oligotrophic species are decrease significantly. It can confirm the organic pollution trend over the lake with correlation of tropic state and organic pollution input. Therefore, the lake tropic state is increased with intensity of self- 
purification. The nutrition type of species in communities of three studied areas demonstrated prevailing of autotrophes nut few species of facultative heterotropes were revealed in the Akshi communities (Fig. $7 d$ ).

We compared bioindication results of the Alakol Lake with diverse NorthKazakhstan area lakes (Barinova et al., 2002; Barinova et al., 2011; Krupa et al., 2016) and find that the lake morphometry play sufficient role in the self-purification processes in the semi-arid climate. Therefore, in followed comparison was included the Alakol Lake and the large lakes and reservoirs in Kazakhstan that were assessed with same methods. So, the Shardara reservoir bioindication was revealed different sources of organic pollution (Barinova and Krupa, 2016, 2017b), which come from the Syr-Darya River as well as the non-point sources of pollution from agricultural fields. The Balkhash Lake bioindicacion also confirm diversity of water pollution sources from fishing and agriculture as well as from the inflowing rivers (Krupa et al., 2014; Krupa et al., 2017a,b,c; Barinova et al., 2017, 2018a,b).

We tried to reveal major factors that influenced algal communities in the Alakol Lake with help of statistical methods. Because the chemical analysis has been not doing, we choosed few biological parameters that influenced algal community. There are Saprobity index $S$ value that reflects organic pollution load and Shannon Index value that demonstrate complication of community structure which is better when Shannon Index is high. Total species richness is also correlated not only with ecosystem health but also reflect better environment for algal community development. So, surface plots were constructed by Distance Weighted Least Squares method for each taxonomic Division in respect of above-mentioned parameters. Fig. 8 represents 3-D surface plots for four taxonomic Divisions species richness. Can be seen, that Cyanobacteria (Fig. 8a), Chlorophyta (Fig. 8b) and Charophyta (Fig. 8c) species were prefer low species richness but complicated communities with high Shannon Index. However, euglenoid species demonstrated opposite tendency (Fig. 8d).

Diatom species are prevail in the lake community, so, distribution of its diversity over same variables can be most important for current analysis. Fig. 9 shows surface plots in which can be seen that diatoms prefer high species rich and low complicated communities (Fig. 9a) that are formed in the low organically polluted waters (Fig. 9b) of the Alakol Lake.

Species richness of other four Divisions distribution in respect of organic pollution reflected by Saprobity index $\mathrm{S}$ show tendency for better grows in high species rich communities in mostly saturated by organic waters in the lake (Fig. 10a-c).

We calculated relationships between algal species in communities of the Alakol Lake and water paramrters with help of CANOCO program in purpose to reveal major factors that influenced algal species development. Fig. 11 represents triplot of calculation the relationships of dominant species (first 10 most abundant taxa in the list) and water temperature, $\mathrm{pH}$, Index $\mathrm{S}$, and Total species richness. Can be seen that species richness and community complication are both have the same influence to algal communities. But, water temperature and organic pollution load has opposite influence. In the upper part of the plot are concentrated three communities from Kamyskala and Akshi areas, which dominated by Chara aspera and Cymbella cymbiformis. These communities have low species richness and therefore have low Shannon Index. It usually happened when charophytes rather developed and forms mats. Other communities are indifferent to studied parameters excluding Nizschia nana in the Kamyskala area, which prefer low temperature waters enriched by organic matter. This conclusion is confirmed by 
Nitzschia nana species-specific ecology that are saprophil, alphamesosaprobiont, mesotroph with species-specific Saprobity index s=3.0.

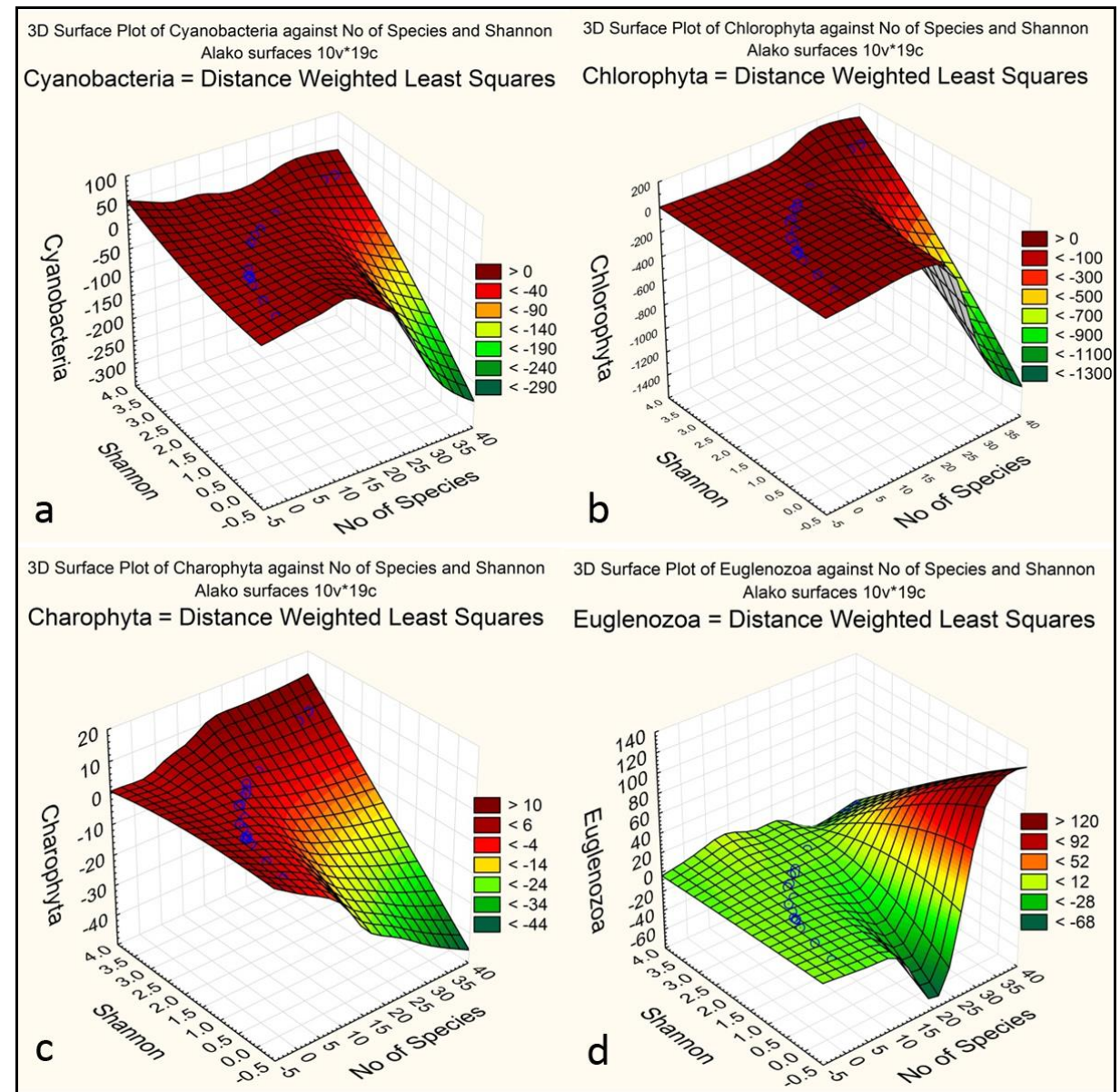

Figure 8. Distribution of algal Divisions species richness over Shannon Index value and Total species richness in communities of the Alakol Lake in 2015-2017. a, Cyanobacteria; b, Chlorophyta; $c$, Charophyta; $d$, Euglenophyta

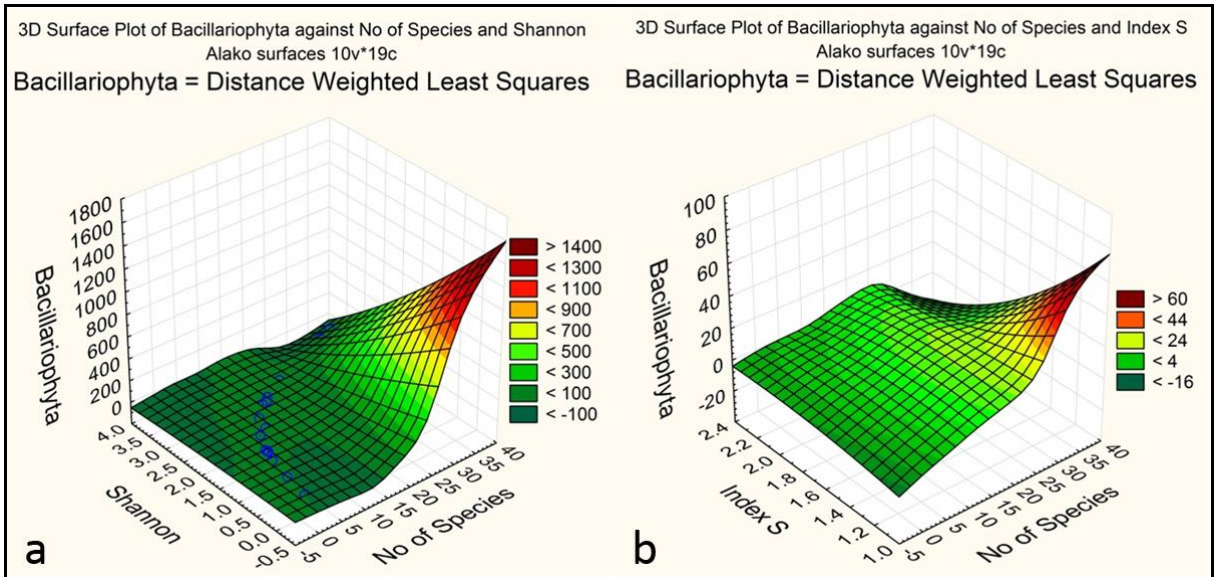

Figure 9. Distribution of Bacillariophyta species richness over Shannon Index (a), Index saprobity (b) value and Total species richness in communities of the Alakol Lake in 2015-2017 


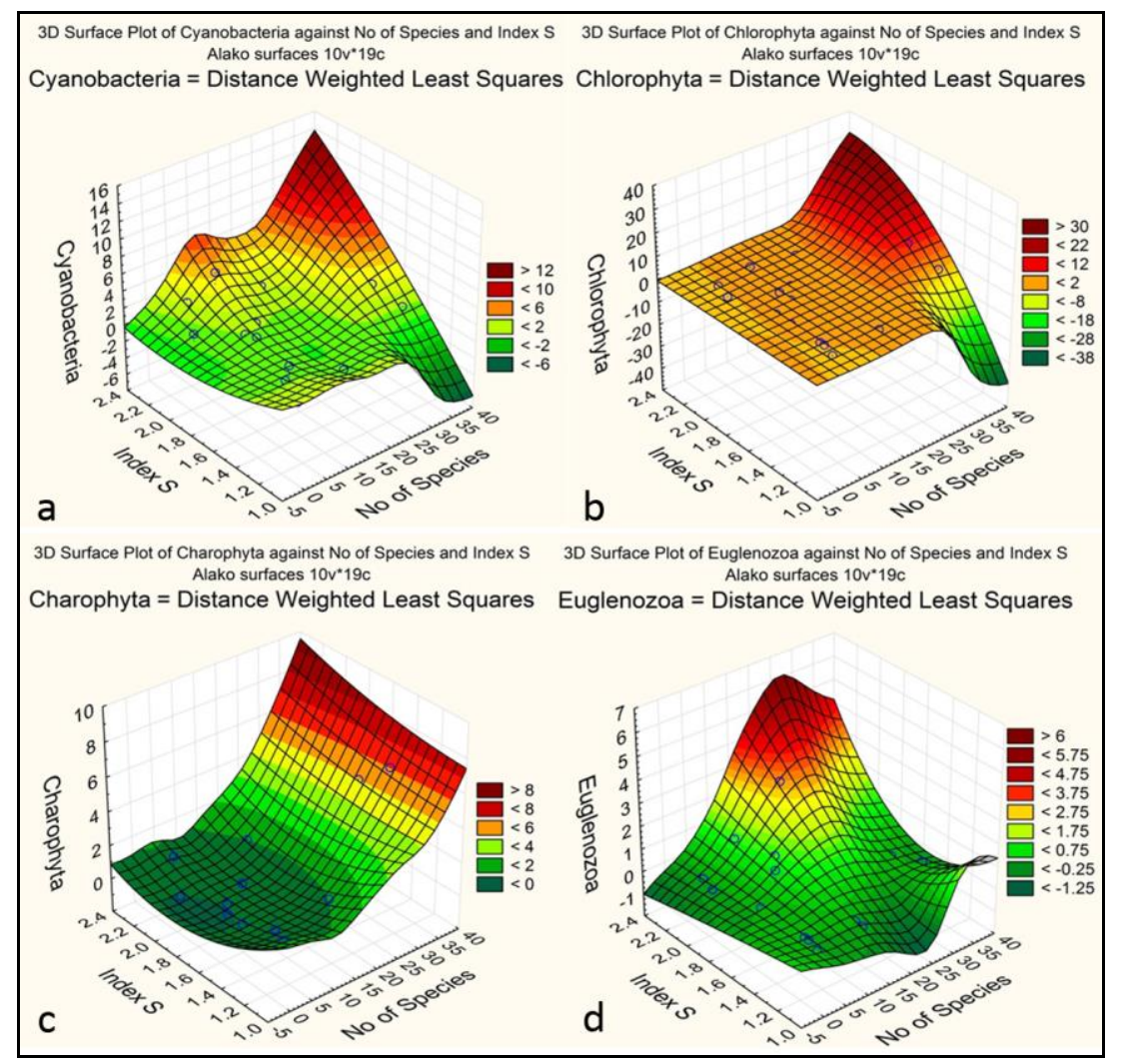

Figure 10. Distribution of algal Divisions species richness over Saprobity index value and Total species richness in communities of the Alakol Lake in 2015-2017. a, Cyanobacteria; $b$, Chlorophyta; c, Charophyta; d, Euglenophyta

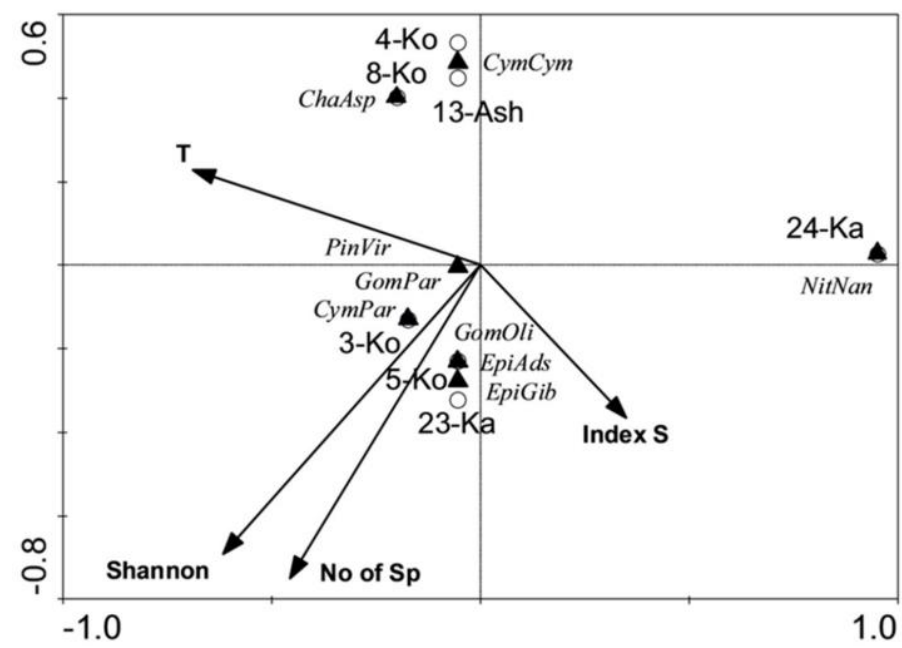

Figure 11. Canonical Correspondence Analysis of dominant algal species in communities and water temperature, organic pollution (as Saprobity index S), complicity of community structure (as Shannon Index), and total species richness in communities of the Alakol Lake in 2015-2017. Abbreviation: Ko, Koktuma area; Ash, Akshi area: Ka, Kamyskala area. ChaAsp, Chara aspera; CymCym, Cymbella cymbiformis; NitNan, Nizschia nana; PinVir, Pinnularia viridis; CymPar, Cymbella parva; EpiAds, Epithemia adnata var. saxonica; EpiGib, Epithemia gibba; PleElo, Pleurosigma elongatum; GomOli, Gomphonema olivaceum; GomPar, Gomphonema parvulum 


\section{Conclusion}

We implemented in the first time ecological assessment of water quality with bioindication methods in the Alakol Lake Natural Reserve. Bioindication of 10 lake water properties was doing with species-specific preferences of 208 indicator species of five taxonomic Divisions of algae collected in summer of 2015-2017 in 21 sampling points along the lake shore. This approach helps us to reveald pollution sources as well as critical variables that influenced water quality in the Alakol Lake Natural Reserve. The prevalence of diatom species allowed us to implement few bioindication systems for conclusion about water properties. In indicator groups were prevaled bentic, temperate water inhabitants, which preferred middle oxygenated and low alkaline water. Indication of water salinity show low concentration of chlorides while total dissolved solids (TDS) of water was rather high due to sulfates. Most important variable such as organic pollution in protected lake was assessed by different indication methods and revealed pollution input in Koktuma and Kamyskala areas. The organic enrichment of the lake water was decrease from northern area Kamyskala to southern Koktuma that means high self-purification properties of the lake ecosystem. In the same time tropic state of the lake and intensity of self-purification increased from north to south. Algal communities were dominated by ten taxa of diatom microalgae in periphyton as well as by macrophyte algae Chara and Nitella. Statistical approach with CCA helps us to reveal major ecosystem properties of three types of algal communities which prefer organically enriched cool water, monodominant community of charophytes, and other indiffer to water properties. It was confirmed by statistically generated surface plots where the most of species preferred low-organically polluted waters with complicated community structure. Bioindication show also that in the lake inhabited two type of really autotrophic communities that preferred oligotrophic and/or meso-eutrophic waters.

Therefore, bioindicational assessment of protected lake is reflect of current status of its water properties and community structure that can be used as references for future monitoring of the Alakol Lake Natural State Reserve.

Acknowledgements. The work was partly supported by Committee of Science, Ministry of Education and Science, Republic of Kazakhstan as well as by the Israeli Ministry of Aliyah and Integration.

\section{REFERENCES}

[1] Barinova, S. (2017a): How to Align and Unify the Cell Counting of Organisms for Bioindication. - International Journal of Environmental Sciences and Natural Resources 2(2): 555585. DOI: 10.19080/IJESNR.2017.02.555585.

[2] Barinova, S. (2017b): On the Classification of Water Quality from an Ecological Point of View. - International Journal of Environmental Sciences and Natural Resources 2(2): 1-8. DOI: 10.19080/IJESNR.2017.02.555581.

[3] Barinova, S. S. (1988): Polymorphism of connective structures in diatom algae. Evolutionary Research. Vavilov's Themes. Vladivostok: Russian Academy of Science, Far East Branch Press, pp. 110-122. (in Russian).

[4] Barinova, S. S., Karlsen, A. G., Solovieva A. A. (2002): Sustainable assessment of some water ecosystems of Kostanai Oblast and west part of North-Kazakhstan Oblast on the hydrochemical and hydrobiological dates. - In: Bragina, T. M., Bragin E. A. (eds.) The 
most important wetlands of North Kazakhstan (Kostsnai Oblast and west part of NorthKazakhstan Oblast), World Wildlife Found Press, Moscow: 39-43. (in Russian).

[5] Barinova, S. S., Medvedeva, L. A., Anissimova, O. V. (2006): Diversity of Algal Indicators in Environmental Assessment. - Pilies Studio, Tel Aviv (in Russian).

[6] Barinova, S. S., Bragina, T. M., Nevo, E. (2009): Algal species diversity of arid region lakes in Kazakhstan and Israel. - Community Ecology 10(1): 7-16. DOI 10.1556/ComEc.10.2009.1.2.

[7] Barinova, S. S., Nevo, E., Bragina, T. M. (2011): Ecological assessment of wetland ecosystems of northern Kazakhstan on the basis of hydrochemistry and algal biodiversity. - Acta Botanica Croatica 70(2): 215-244. DOI 10.2478/v10184-010-0020-7.

[8] Barinova, S., Krupa, E. (2016): Critical environmental factors for photosynthetic organisms of the Shardara Reservoir, Kazakhstan. - Bulletin of Advanced Scientific Research 2(5): 17-27.

[9] Barinova, S. S., Krupa, E. G. (2017a): Diversity and Ecology of Periphytonic Algae in the Arys River Basin, Kazakhstan. - Journal of Ecology and Natural Resources 1(1): 114.

[10] Barinova, S., Krupa, E. (2017b): Bioindication of ecological state and water quality by phytoplankton in the Shardara Reservoir, Kazakhstan. - Environment and Ecology Research 5: 73-92. DOI: 10.13189/eer.2017.050201.

[11] Barinova, S., Krupa, E., Kadyrova, U. (2017): Spatial dynamics of species richness of phytoplankton of Lake Balkhash (Kazakhstan) in the gradient of abiotic factors. Transylvanian Review of Systematical and Ecological Research. "The Wetlands Diversity" 19(2): 1-18.

[12] Barinova, S., Krupa, E., Romanova, S. (2018a): The role of planktonic algae in the ecological assessment of storage-reservoirs of the Ili-Balkhash basin. - Transylvanian Review of Systematical and Ecological Research, "The Wetlands Diversity" 20(2): 1-14. DOI: 10.2478/trser-2018-0001.

[13] Barinova, S., Krupa, E., Tsoy, V., Ponamareva, L. (2018b): The application of phytoplankton in ecological assessment of the Balkhash Lake (Kazakhstan). - Applied Ecology and Environmental Research 16(3): 2089-2111. DOI: http://dx.doi.org/10.15666/aeer/1603_20892111.

[14] Berezovikov, N. (2006): Alakol State Nature Reserve. - In: Yaschenko, R. V. (ed.). Reserves of Central Asia and Kazakhstan. Protected natural areas of Central Asia and Kazakhstan. Issue. 1. Almaty, Tethys, pp. 12-13. (in Russian).

[15] Cavalier-Smith, T. (2004): Only six kingdoms of life. - Proceedings of the Royal Society, London, B 271: 1251-1262.

[16] Freshwater ecoregions of the world (FEOW). - Available from: http://feow.org/ [accessed 20 June 2018].

[17] Guiry, M. D., Guiry, G. M. (2018): AlgaeBase, World-wide electronic publication. National University of Ireland Press, Galway. [accessed 20 June 2018].

[18] Jiyenbekov, A, Barinova, S., Bigaliev, A., Nurasov, S., Sametova, E. (2018): The first evidence about the algae of the protected Alakol lake (Kazakhstan) and their floral analysis. - Bulletin of Moscow society of naturalists, biological series. (in Press).

[19] Krupa, E. G., Amirgalieva, N. A., Lopareva, T. Ya., Isaeva, A. K., Bimanbaeva, B. B. (2010): Zooplankton of the Lake Alakol and its distribution depending on mineralization and chemical composition of water. - Bulletin of the Kazakh National University AlFarabi, biological series, Almaty 1(43): 96-100. (in Russian).

[20] Krupa E. G., Barinova S. S., Romanova S. M., Malybekov A. B. (2016): Hydrobiological assessment of the high mountain Kolsay lakes (Kungey Alatau, Southeastern Kazakhstan) ecosystems in climatic gradient. - British Journal of Environment and Climate Change 6(4): 259-278.

[21] Krupa E. G., Barinova S. S., Isbekov K. B., Tsoy V. N., Assylbekova S. Z., Sharipova O. A. (2017a): Influence of chemical water composition on spatial distribution of 
phytoplankton in the Balkhash Lake (Kazakhstan). - Research Journal of Pharmaceutical, Biological and Chemical Sciences 8(5): 396-411.

[22] Krupa, E. G., Barinova, S. S., Tsoy, V. N., Lopareva, T. Y., Sadyrbaeva, N. N. (2017b): Spatial analysis of hydrochemical and toxicological variables of the Balkhash Lake, Kazakhstan. - Research Journal of Pharmaceutical, Biological and Chemical Sciences 8(3): 1827-1839.

[23] Krupa, E. G., Barinova, S. S., Tsoy, V. N., Sadyrbaeva, N. N. (2017c): Formation of phytoplankton of Lake Balkhash (Kazakhstan) under the influence of major regionalclimatic factors. - Advances in Biology and Earth Sciences 2(2): 204-213.

[24] Krupa, E. G., Barinova, S. S., Amirgaliyev, N. A., Issenova, G., Kozhabayeva, G. (2017d): Statistical approach to estimate the anthropogenic sources of potentially toxic elements on the Shardara Reservoir (Kazakhstan). - MOJ Ecology and Environmental Sciences 2(1): 1-8. DOI: 10.15406/mojes.2017.02.00012.

[25] Krupa, E. G., Barinova, S. S., Assylbekova, S. Z., Isbekov, K. B. (2018a): Structural indicators of zooplankton in the Shardara Reservoir (Kazakhstan) and the main influencing factors. - Turkish Journal of Fisheries and Aquatic Sciences 18: 659-669. DOI: 10.4194/1303-2712-v18_5_02.

[26] Krupa, E. G., Barinova, S. S., Isbekov, K. B., Assylbekova, S. Z. (2018b): The use of zooplankton distribution maps for assessment of ecological status of the Shardara reservoir (Southern Kazakhstan). - Ecohydrology and Hydrobiology 18(1): 52-65. https://doi.org/ 10.1016/j.ecohyd.2017.10.001.

[27] Krupa, E., Slyvinskiy, G., Barinova, S. (2014): The Effect of Climatic Factors on the Long-term Dynamics of Aquatic Ecosystem of the Balkhash Lake (Kazakhstan, Central Asia). - Advanced Studies in Biology 6(3): 115-136.

[28] Novakovsky, A. B. (2004): Abilities and base principles of program module "GRAPHS." - Scientific Reports of Komi Scientific Center, Ural Division of the Russian Academy of Sciences 27: 1-28.

[29] Ter Braak, C. J. F., Šmilauer, P. (2002): CANOCO reference manual and CanoDraw for Windows user's guide: software for Canonical Community Ordination (version 4.5). Microcomputer Power Press, Ithaca.

[30] Watanabe, T., Asai, K., Houki, A. (1986): Numerical estimation to organic pollution of flowing water by using the epilithic diatom assemblage - Diatom Assemblage Index (DAIpo). - Science of Total Environment 55: 209-218. 